Pacific Journal of Mathematics

FIVE THEOREMS ON MACAULAY RINGS

HOCHSTER AND LOUIS JACKSON RATLIFF, JR. 


\title{
FIVE THEOREMS ON MACAULAY RINGS
}

\author{
M. Hochster AND L. J. RATLIFF, JR.
}

The first three theorems concern localizations of a Noetherian ring such that the localization is a Macaulay ring, and the other two theorems give some necessary and sufficient conditions for certain Rees rings and form rings of a Noetherian ring to be locally Macaulay. Numerous consequences of the theorems are proved.

1. Introduction. Macaulay rings have been extensively studied, and numerous properties of such rings are known. It is the purpose of this paper to add to the knowledge of such rings (and Noetherian ring theory in general) by proving five theorems in this area, and to derive some consequences of these theorems.

Most of the results in $\S 2$ follow quite readily from the first theorem, Theorem 2.2. Among the corollaries of this theorem, are the following, where $E$ is a finite module over a Noetherian ring $R$ such that $E_{Q} \neq(0)$, for some $Q \in \operatorname{Spec} R$. The Macaulay locus of $E$ (that is, the $Q \in \operatorname{Spec} R$ such that $E_{Q}$ is Macaulay) is open in the $V S$ topology (Definition 2.3) on Spec $R$ (2.4.1). If $P \in \operatorname{Spec} R$ and height $P /($ Ann $E)>1$, then there exist infinitely many $p \in$ Spec $R$ such that Ann $E \subset p \subset P$, height $P / p=1$, and $E_{p}$ is Macaulay (2.5.2). $\operatorname{Rad}(\operatorname{Ann} E)=$ $\cap\left\{p \in \operatorname{Spec} R ; E_{p}\right.$ is Macaulay and $p$ is a $G$-ideal $\}$ (that is, the $G$-ideals of the Macaulay locus are Zariski dense in the set of prime ideals containing Ann $E$ ) (2.12). (An important special case is when $E=R$ is a Hilbert ring (2.17).) If $E_{Q}$ is Macaulay and $P \in \operatorname{Spec} R$ is such that $Q \subset P$ and height $P / Q=d$, then, for $i=0,1, \cdots, d-1, Q=\cap$ $\left\{p \in \operatorname{Spec} R ; Q \subseteq p \subset P, E_{p}\right.$ is Macaulay, height $p=$ height $Q+i$, height $P / p=d-i$, and $i=$ height $p / Q\}$ (2.19). Also these last intersectors can be adjusted to not being contained in a finite set of prime ideals which don't contain $P(2.21)$, and to not containing a given element not in $Q$ (2.23). Then this section is closed by proving a result, Proposition 2.26, which implies that, in a local ring $(R, M)$, there exists a system of parameters $b_{1}, \cdots, b_{a}$ such that, if $P \neq M$ is a prime ideal in $R$ such that $b_{1}, \cdots, b_{j} \in P$, for some $j<a$, then the images in $R_{P}$ of $b_{1}, \cdots, b_{j}$ are an $R_{P}$-sequence and, if $P$ is a minimal prime divisor of $\left(b_{1}, \cdots, b_{j}\right) R$, then, for $i=0,1, \cdots, j,\left(b_{1}, \cdots, b_{i}\right) R_{P}$ is a primary ideal (by (2.26.3) and (2.27.1)).

In $\S 3$, two theorems concerning a finitely generated ring $A$ over a Noetherian ring $R$ are proved. The first, Theorem 3.9, shows in particular that, if $A$ is an integral domain and $R$ satisfies the condition: $(0)=\cap\left\{M ; M\right.$ is a maximal ideal in $R$ and $R_{M}$ is Macaulay $\}$ : then $A$ 
does. The second, Theorem 3.12, gives a necessary and sufficient condition for $A$ to satisfy this condition, when $R$ is semilocal and altitude $R>1$. The theorem implies that most non-integral extensions of $R$ do satisfy the condition and, if $R$ is integrally closed, then every proper extension domain of $R$ satisfies the condition (Corollary 3.13).

The last two theorems are proved in $\S 4$. The first of these and all its corollaries are closely related to (and/or are generalizations of) the results in [11]. Specifically, it is proved in [11] that the Rees ring $\mathscr{R}(A, Y)$ of a locally Macaulay ring $A$ with respect to an ideal $Y$ generated by an $A$-sequence is locally Macaulay, and some related results are obtained. In $\S 4$, a number of necessary and sufficient conditions are given for the Rees ring $\mathscr{R}=\mathscr{R}(R, B)$ to be locally Macaulay, where $\left(R ; M_{1}, \cdots, M_{e}\right)$ is a semi-local ring such that height $M_{i}=$ altitude $R=a(i=1, \cdots, e)$ and $B$ is an ideal in $R$ such that Rad $B=\cap M_{i}$. Namely, the following conditions are equivalent: (1) $\mathscr{R}$ is locally Macaulay; (2) The form ring $\mathscr{F}$ of $R$ with respect to $B$ is locally Macaulay; (3) $\mathscr{R}_{\left(\mathscr{R} \sim \cup \mathscr{M}_{i}\right)}\left(\right.$ resp., $\left.\mathscr{F}_{\left(\sim \sim \cup \mathscr{N}_{i}\right)}\right)$ is a Macaulay ring, where the $\mathscr{L}_{i}$, $\left(\right.$ resp., $\left.\mathscr{N}_{i}\right)(i=1, \cdots, e)$ are the maximal homogeneous ideals in $\mathscr{R}$ (resp., $\mathscr{F}$ ); (4) $R$ is Macaulay and there exists a system of parameters $y_{1}, \cdots, y_{a}$ in $R$ such that, for each $j=1, \cdots$, $a$ and for all $n,\left(y_{1}, \cdots, y_{j}\right) R \cap B^{n}=\sum_{1}^{j} y_{i} B^{n-d_{i}}$, where $y_{i} \in B^{d_{i}}$, $\notin B^{d_{i}+1}$; (5) $R$ is Macaulay and there exists a positive integer $g$ and a system of parameters $z_{1}, \cdots, z_{a}$ contained in $B^{g}$ such that, for each $j=1, \cdots$, $a$ and for all $n \geqq g,\left(\left(z_{1}, \cdots, z_{j}\right) R\right) \cap B^{n}=\left(z_{1}, \cdots, z_{j}\right) B^{n-g}$ (by (4.4), (4.6), and (4.11)). Further, if $\mathscr{R}$ is locally Macaulay, then following hold: (a) Given any finite number (say, $s$ ) of prime ideals $P_{i}$ in $R$ which have the same height (say, $k$ ), there exists an $R$-sequence $y_{1}, \cdots, y_{k}$ contained in $\cap P_{i}$ such that, for each $j=1, \cdots, k$ and for all $n,\left(y_{1}\right.$, $\left.\cdots, y_{j}\right) R \cap B^{n}=\sum_{1}^{j} y_{i} B^{n-d_{i}}$ with $d_{i}$ as in (4) (Corollary 4.5); (b) $\mathscr{R}(R$, $B^{m}$ ) is locally Macaulay, for all $m>0$ (Corollary 4.8.1); and, (c) For all $m>0, R$ and $R\left[B^{m} / b\right]$ are locally Macaulay for each nonzero-divisor $b \in B^{m}$ (by 4.8.2) and [11, Theorem 3.8]). Also, (a) - (c) hold when $B$ is a power of an ideal generated by an $R$-sequence (Corollary 4.9). The last theorem (4.11) shows that (1) - (3) above are equivalent for an arbitrary j.deal $B$ in a Noetherian ring such that $B$ is contained in the Jacobson radical of $R$, and each of (1) - (3) implies (c) for $m=1$. Some related information is given in Propositions 4.10 and 4.12.

2. Macaulay localizations. The terminology in this article is, in general, the same as that in [8]. However, to keep the article reasonably self-contained, a number of definitions will be given. In particular, if $R$ is a ring and $E$ is an $R$ module, then the (ordered sequence of) elements $b_{1}, \cdots, b_{n}$ in $R$ is an $E$-sequence in case: $\left(b_{1}, \cdots\right.$, $\left.b_{n}\right) E \neq E$; and, for $i=1, \cdots n, b_{i}$ isn't a zero-divisor on $E /\left(b_{1}, \cdots, b_{i-1}\right) E$. 
(As usual, the ideal generated by the empty set is defined to be the zero ideal, and $b \in R$ is a zerodivisor on an $R$-module $E^{*}$ in case $b x=$ 0 , for some nonzero $x \in E^{*}$.) Ass $E=\{p \in \operatorname{Spec} R ;(0):(x)=p$, for some nonzero $x \in E\}, \quad A n n E=(0): E=\{r \in R ; r E=(0)\}, \operatorname{Dim} E=$ altitude $R /($ Ann $\mathrm{E})$, and Prof $E$ is the length of a maximal $E$-sequence. If $R$ is a local (Noetherian) ring and $E$ is a finite $R$-module, then $E$ is a Macaulay $R$-module in case either $E=(0)$ or $E \neq(0)$ and $\operatorname{Dim} E=$ Prof $E$. (However, in this paper, the statement that $E$ is Macaulay will always mean $E \neq(0)$ and $E$ is Macaulay.) A local ring $R$ is a Macaulay local ring in case $R$ is a Macaulay $R$-module (that is, there exists a system of parameters in $R$ which is an $R$-sequence). A Noetherian ring $R$ is said to be a locally Macaulay ring in case $R_{P}$ is a Macaulay local ring, for all prime ideals $P$ in $R$; and $R$ is said to be a Macaulay ring in case $R$ is a locally Macaulay ring and height $M=$ altitude $R$, for all maximal $M$ in $R$.

Many basic properties of Macaulay rings and Macaulay modules will be used implicitly throughout this paper. These properties can be found in [2, Chapter $0, \S 16.5],[5$, Chapter 3], and [8, §25].

The following lemma is of basic importance in this section. (It should be noted that, in the lemma, $\mathscr{P}$ may be a finite set.)

Lemma 2.1. Let $Q$ be a prime ideal in a Noetherian ring $R$, let $B_{1}, \cdots, B_{h}$ be ideals in $R$ which are contained in $Q$, let $\mathfrak{P}_{j}$ be the set of prime divisors of $B_{j}$, and let $\mathscr{P}$ be the set of prime ideals $p$ in $R$ such that $Q \subset p$ and height $p / Q=1$. Then there are at most $a$ finite number of $p \in \mathscr{P}$ such that either $p$ contains an ideal $P \in \cup_{0}^{h} \Re_{j}$ such that $P \notin Q$ or height $p>$ height $Q+1$.

Proof. It is known [6] that there are only finitely many $p \in \mathscr{P}$ such that height $p>$ height $Q+1$. Also, at most a finite number of $p \in \mathscr{P}$ can contain a fixed ideal which isn't contained in $Q$ (since $R / Q$ is Noetherian). Hence, since $\bigcup_{0}^{h} \Re_{j}$ is a finite set, the lemma follows.

Most of the results in this section follow quite readily from the following theorem.

Theorem 2.2. Let $R, Q$, and $\mathscr{P}$ be as in (2.1), and let $E$ be a finite $R$-module. If $E_{Q}$ is Macaulay, then there are at most a finite number of $p \in \mathscr{P}$ such that $E_{p}$ is not Macaulay.

Proof. Let $\operatorname{Dim} E_{Q}=h$, and let $b_{1}, \cdots, b_{h}$ be elements in $Q$ such that their images in $R_{Q}$ are an $E_{Q}$-sequence and such that height $B_{j}=j(j=0,1, \cdots, h)$, where $B_{j}=\left(b_{1}, \cdots, b_{j}\right) R$. Then $\bigcup_{0}^{h}$ Ass $\left(E / B_{j} E\right)$ 
is a finite set of prime ideals in $R$, so, by (2.1) only finitely many ideals in $\mathscr{P}$ can contain an element in this set which isn't contained in $Q$ or can have height greater than height $Q+1$. Omitting these, it is readily checked that, for all other $p \in \mathscr{P}, E_{p}$ is Macaulay.

Definition 2.3. Let $R$ be a Noetherian ring, and let $U \subseteq \operatorname{Spec} R$. Then $U$ is said to be VS-open in case the following condition holds: If $Q \in U$, then $U$ contains all but finitely many prime ideals $p$ in $R$ such that $Q \subset p$ and height $p / Q=1$. It is clear that the $V S$-open sets are the open sets of a topology on Spec $R$ called the very strong topology (VS topology) on Spec $R$.

A subset of Spec $R$ which is either open or closed in the Zariski topology on Spec $R$ is VS-open (for Zariski open, see (2.1)). Also, using (2.1) it is readily seen that the VS topology is Hausdorff and totally disconnected.

REMARK 2.4. 2.4.1. It follows from (2.2) that if $Q \in \operatorname{Spec} R$ is such that $E_{Q}$ is Macaulay, then $\left\{p \in \operatorname{Spec} R ; Q \subseteq p\right.$ and $E_{p}$ is Macaulay $\}$ is $V S$-open. Thus, since, for each minimal prime ideal $Q \in \operatorname{Ass} E, E_{Q}$ is Macaulay (since $\operatorname{Dim} E_{Q}=0$ ), (2.2) asserts that the Macaulay locus of $E$ (that is, the set of $Q$ in Spec $R$ such that $E_{Q}$ is Macaulay) is VS-open.

2.4.2. In [3, pp. 162-163], the question of whether the Macaulay locus of $R$ is always open in the Zariski topology was raised. An affirmative answer is given there for homomorphic images of locally Macaulay rings [3, (6.11.8)]. However, in [1, Proposition 3.5], an example is given to show that, in general, the answer is "no".

Corollary 2.5. Let $E$ be a finite module over a Noetherian ring $R$, let $P$ be a prime ideal in $R$ such that $A n n E \cong P$, and let height $P /($ Ann $E)=h>1$.

2.5.1. If $Q$ is a prime ideal in $R$ such that $Q \subset P$, height $P / Q>1$, and $E_{Q}$ is Macaulay, then, for all but a finite number of prime ideals $p$ in $R$ such that $Q \subset p \subset P$ and height $p / Q=1, E_{p}$ is Macaulay.

2.5.2. There exist infinitely many prime ideals $p$ in $R$ such that Ann $E \subset p \subset P$, height $P / p=1$, and $E_{p}$ is Macaulay.

Proof. On localizing at $P,(2.5 .1)$ follows immediately from (2.2), and (2.5.2) follows from successive applications of (2.5.1) with $Q$ a minimal prime ideal in Ass $E$. 
It follows from (2.19) below that in (2.5.2) infinitely many of the ideals $p$ satisfy height $p /(\operatorname{Ann} E)=h-1$.

Some further corollaries to (2.2) will be given below. However, before this, some topological remarks will be given. The following lemma sets the stage for these remarks. (In the lemma, $R_{c}$ is the ring $R_{S}$, where $S=\left\{c^{i} ; i \geqq 0\right\}$.)

LEMma 2.6. The following statements are equivalent for a prime ideal $Q$ in a Noetherian ring $R$ :

2.6.1. The set of $P$ in Spec $R$ containing $Q$ is finite.

2.6.2. Depth $Q \leqq 1$ and $R / Q$ is a semi-local ring.

2.6.3. There exists an element $c \in R, \notin Q$, such that $Q R_{c}$ is a maximal ideal in $R_{c}$.

2.6.4. There exists a finitely generated extension ring $R^{\prime}$ of $R$ which contains a maximal ideal $M$ such that $M \cap R=Q$.

2.6.5. $Q$ is isolated in the VS topology on Spec R.

Proof. (2.6.2) $\Leftrightarrow(2.6 .4)$ follows from [8, (14.10)], and the rest of the proof is straightforward.

Definition 2.7. A prime ideal in a Noetherian ring $R$ which satisfies the equivalent conditions of (2.6) is a $G$-ideal.

It is easy to see that every prime ideal in $R$ is an intersection of $G$-ideals. Also, the set of $G$-ideals is the least class of prime ideals in $R$ which has this property. Concerning $G$-ideals in an arbitrary commutative ring with unit element, see [5, pp. 12-21].

Definition 2.8. Let $R$ be a Noetherian ring, and let $B \cong \operatorname{Spec} R$. $B$ is said to be big in case the following condition holds: If $Q \in B$ and $Q$ isn't a $G$-ideal, then the set of $p \in B$ such that $Q \subset p$ and height $p / Q=1$ is an infinite set.

REMARK 2.9. It is readily seen that VS-open sets are big. Also, if $U$ is $V S$-open and $B$ is big, then $U \cap B$ is big.

For an example of a big set which may not be VS-open, see (2.18) and the comment which follows it.

The notion of bigness is worth isolating because of the following lemma and corollaries.

Lemma 2.10. If $R$ is Noetherian and $B \cong$ Spec $R$ is big, then, for each $Q \in B, Q=\cap\{P \in B ; Q \subseteq P$ and $P$ is a $G$-ideal $\}$.

Proof. Suppose not, and let $Q \in B$ be maximal for which the lemma doesn't hold. Then $Q$ isn't a $G$-ideal, so, since $B$ is big, $\mathscr{P}=$ $\{P \in B ; Q \subset P$ and height $P / Q=1\}$ is an infinite set, hence $Q=\cap\{P$; 
$P \in \mathscr{P}\}$. By maximality of $Q$, a contradiction obtains, since each $P \in \mathscr{P}$ is the intersection of the $G$-ideals in $B$ which contain it.

CoRollary 2.11. Let $R$ be a Noetherian ring, $Q$ a prime ideal in $R$, and $E$ a finite $R$-module. If $E_{Q}$ is Macaulay, then $Q=\cap\{p \in$ Spec $R ; Q \subseteq p, E_{p}$ is Macaulay, and $p$ is a G-ideal\}.

Proof. Let $\mathscr{P}=\left\{p \in \operatorname{Spec} R ; Q \subseteq p\right.$ and $R_{p}$ is Macaulay $\}$. Then $\mathscr{P}$ is $V S$-open (2.4.1), hence big (2.9), and so the corollary follows from (2.10).

The assertion in (2.11) that $Q=\cap p_{\lambda}$ says, geometrically, that the $p_{\lambda}$ are Zariski dense in the locus of (set of prime ideals containing) $Q$. Thus, this section of the paper is, in two related senses, concerned with the fact that prime ideals $Q$ such that $E_{Q}$ is Macaulay are plentiful: openness in the VS topology, and density in various closed sets in the Zariski topology.

If $R$ is a Noetherian ring of altitude one or a Noetherian domain of altitude two, then $R_{P}$ is Macaulay, for all non-maximal prime ideals $P$ in $R$-even if $R$ isn't Macaulay. On the other hand, if $R$ is a local ring with a prime ideal $Q$ such that depth $Q \geqq 2$ and $R_{Q}$ isn't Macaulay, then there are infinitely many prime ideals $p$ in $R$ such that $Q \subset p$ and, for each such $p, R_{p}$ isn't Macaulay. Even so, the following corollary shows, with $R=E$, that, for an arbitrary Noetherian ring $R$ of altitude greater than one, there are infinitely many prime ideals $P$ in $R$ such that depth $P \leqq 1$ and $R_{P}$ is Macaulay. (However, as already noted, the Macaulay locus of $R$ need not be Zariski-open [1, Proposition 3.5].)

Corollary 2.12. If $R$ is a Noetherian ring, and $E \neq(0)$ is a finite $R$-module, then Rad $($ Ann $E)=\cap\left\{p \in S p e c R ; E_{p}\right.$ is Macaulay and $p$ is a G-ideal $\}$. Hence, $\left\{p \in S p e c R ; R_{p}\right.$ is Macaulay and $p$ is a $G$-ideal\} is Zariski dense in Spec R.

Proof. For each minimal prime ideal $q$ in Ass $E, E_{q}$ is Macaulay. Hence the corollary follows from (2.11), since $\operatorname{Rad}(\operatorname{Ann} E)=\cap\{q ; q \in$ Ass $E\}$.

Two special cases of the following corollary will be given in (2.14) and $(2.20)$.

CoROLlary 2.13. Let $I$ and $J$ be ideals in a Noetherian ring $R$ such that $I \subseteq R a d J$, and let $E$ be a finite $R$-module. If $E_{Q} / I E_{Q}$ is Macaulay, for each minimal prime divisor $Q$ of $J$, then $R a d J=\cap$ $\left\{p ; p\right.$ is a G-ideal in $R, J \subseteq p$, and $E_{p} / I E_{p}$ is Macaulay $\}$. 
Proof. Reducing to the case $I=(0)$, the result follows from (2.11).

Corollary 2.14. For each ideal $J$ in a Noetherian ring $R$, Rad $J=\cap\left\{p ; p\right.$ is a G-ideal in $R, J \leqq p$, and $R_{p} / J R_{p}$ is Macaulay $\}$.

Proof. This follows from (2.13) with $I=J$ and $E=R$.

REMARK 2.15. 2.15.1. It follows from (2.14) and [5, Theorem 156] that, if $c$ is a nonunit regular element in $R$, then $\operatorname{Rad} c R=\cap$ $\left\{p ; p\right.$ is a $G$-ideal in $R, c \in p$, and $R_{p}$ is Macaulay\}. A similar statements holds for $R$-sequences of length greater than 1 .

2.15.2. In the case $J=Q$ is prime and $R_{Q}$ is Macaulay, (2.11) and (2.14) give an interesting comparison of $Q$ expressed as an intersection of $G$-ideals in $R$. In particular, such a comparison holds for each minimal prime ideal in $R$; and also for each height one prime ideal in $R$, if $R$ is an integral domain. Also, such a comparison holds for a radical ideal $I$ in $R$ such that $R_{p}$ is Macaulay, for each prime divisor $p$ of $I$.

Definition 2.16. A Hilbert ring is a ring $R$ such that each prime ideal in $R$ is the intersection of the maximal ideals in $R$ which contain it.

It is clear by the definition that a factor ring of a Hilbert ring is a Hilbert ring, and it is known [5, Theorem 31] that a finite extension ring of a Hilbert ring is a Hilbert ring.

Corollary 2.17. If $R$ is a Noetherian Hilbert ring and $(0) \neq E$ is a finite R-module, then Rad (Ann $E)=\cap\{M \in$ Spec $R ; M$ is a maximal ideal and $E_{M}$ is Macaulay\}.

Proof. The only $G$-ideals in $R$ are the maximal ideals in $R$, hence the corollary follows from (2.12).

If $P$ is a prime ideal in $R$ and $c$ is a non-nilpotent element in $P R_{P}$, then $\left(R_{P}\right)_{c}$ is a Hilbert ring [4, (10.5.8)]. Using this and (2.17), an alternate proof of (2.5.2) is readily obtained.

It will be seen in (3.3) that in some rings which are neither Hilbert, nor Macaulay, $\operatorname{Rad} R$ is the intersection of the maximal ideals $M$ in $R$ such that $R_{M}$ is Macaulay.

To generalize (2.5.1) and to derive some further corollaries to (2.2), the following lemma is needed.

Lemma 2.18. Let $Q \subset P$ be prime ideals in a Noetherian ring $R$ such that height $P / Q>1$, and let $\mathscr{P}=\{p \in \operatorname{Spec} R ; Q \subset p \subset P\}$. Then 
there are infinitely many $p \in \mathscr{P}$ such that height $p=$ height $Q+1$ and height $P / p=$ height $P / Q-1$.

Proof. In $R_{P} / Q_{P}$ each nonzero non-unit has depth equal to height $P / Q-1$ and has only a finite number of minimal prime divisors, at least one of which must have depth equal to height $P / Q-1$. Hence, since at most a finite number of $p \in \mathscr{P}$ have height greater than height $Q+1$ [6], the conclusion follows.

It follows from (2.18) that the set of prime ideals $p$ between two prime ideals $Q \subset P$ in a Noetherian ring $R$ such that height $p=$ height $Q+$ height $p / Q$ and height $P / p=$ height $P / Q$-height $p / Q$ is big in Spec $R_{P}$ (where Spec $R_{P} \subseteq$ Spec $R$ in a natural way). Using [8, Example 2, pp. 203-205] an example can be given in which the set isn't $V S$-open.

Up to now we've seen that if $E_{Q}$ is Macaulay and $Q$ isn't a $G$ ideal, then $Q=\cap p_{\lambda}$, where $E_{p_{\lambda}}$ is Macaulay and either height $p_{\lambda}=$ height $Q+1$ or where the $p_{\lambda}$ are $G$-ideals. The intermediate cases are given in the following corollary.

Corollary 2.19. Let $R$ be a Noetherian ring, let $Q \subset P$ be prime ideals in $R$ such that height $P / Q=d$, and let $E$ be a finite $R$-module. If $E_{Q}$ is Macaulay, then, for each $i=0,1, \cdots, d-1, Q=\cap\{p ; p \in$ $\left.\mathscr{P}_{i}\right\}$, where $\mathscr{P}_{i}=\left\{p \in S p e c R ; Q \subseteq p \subset P, E_{p}\right.$ is Macaulay, height $p=$ height $Q+i$, height $P / p=d-i$, and $i=$ height $p / Q\}$.

Proof. This follows from successive applications of (2.5.1) and (2.18).

(2.19) Holds, with $E=R$, for each minimal prime ideal $Q$ in $R$ (and for all but a finite number of height one prime ideals $Q$ in $R$ ) with $P$ a maximal ideal in $R$ such that $Q \subset P$. If, moreover, depth $Q=d<\infty$, then $P$ may be chosen such that height $P / Q=d$.

(2.20) shows that in (2.19) the case $i=d$ can be included when $R$ is a Hilbert ring.

Corollary 2.20. Let $E$ be a finite $R$-module and let $Q$ be a prime ideal in a Noetherian Hilbert ring $R$ such that $E_{Q}$ is Macaulay. Then $Q=\cap\{M ; M \in \mathscr{S}\}$, where $\mathscr{S}=\{M ; M$ is a maximal ideal in $R, Q \subseteq$ $M$, and $E_{M}$ is Macaulay\}. Moreover, if depth $Q=d<\infty$, then $Q=$ $\cap\{M ; M \in \mathscr{S}$ and height $M=$ height $Q+d\}$.

Proof. The first statement follows from (2.13) with $I=(0)$ and $J=Q$. For the second statement, if $d=0$, then the conclusion is obvious. If $d>0$, then let $\mathscr{P}=\{p \in \operatorname{Spec} R ; Q \subset p$, height $p=$ height $Q+1$, 
and depth $p=d-1\}$. Then $\mathscr{P}$ is an infinite set (if $d>1$, by (2.18); if $d=1$, by [6], since $R$ is Hilbert), hence $\mathscr{P}^{\prime}=\left\{p \in \mathscr{P} ; E_{p}\right.$ is Macaulay $\}$ is an infinite set, by (2.2). Therefore, $Q=\cap\left\{p ; p \in \mathscr{P}^{\prime}\right\}$. Since each $p \in \mathscr{P}^{\prime}$ satisfies depth $p=d-1$, the corollary follows by induction on $d$.

Corollary 2.21. Let $R$ and $E$ be as in (2.20), let $Q \subset P$ be prime ideals in $R$ such that height $P / Q>1$ and $E_{Q}$ is Macaulay, and let $N_{1}, \cdots, N_{g}$ be prime ideals in $R$ such that $P \nsubseteq \cup N_{j}$. Then, for $i=$ $1, \cdots, d-1, Q=\cap\left\{p ; p \in \mathscr{P}_{i}\right\}, \quad$ where $\mathscr{P}_{i}=\{p \in$ Spec $R ; Q \subset p \subset P$, height $p=$ height $Q+i$, height $P / p=$ height $P / Q-i, i=$ height $p / Q, p \nsubseteq \cup N_{j}$, and $E_{p}$ is Macaulay\}.

Proof. If it can be proved that $Q=\cap\left\{p ; p \in \mathscr{P}_{1}\right\}$, then the result for $i>1$ readily follows from (2.19), so only the case $i=1$ will be considered. Let $\mathscr{P}^{\prime}$ be the finite set of prime ideals $p$ in $R$ such that $Q \subset p \subset P$ and height $p / Q=1<$ height $p$ - height $Q$ [6]. Let $b_{1}, \cdots, b_{h} \in Q$ such that their images in $R_{Q}$ are a maximal $E_{Q}$-sequence and such that height $\left(b_{1}, \cdots, b_{j}\right) R=j(j=0,1, \cdots, h)$. Let $\mathscr{D}_{j}$ be the set of prime ideals in Ass $E /\left(b_{1}, \cdots, b_{j}\right) E$ which don't contain $P$, and let $\mathscr{P}^{*}=\left(\bigcup_{0}^{h} \mathscr{D}_{j}\right) \cup \mathscr{P}^{\prime} \cup\left\{N_{1}, \cdots, N_{g}\right\}$. Then $\mathscr{P}^{*}$ is a finite set of prime ideals in $R$ and $P \nsubseteq \cup\left\{q ; q \in \mathscr{P}^{*}\right\}$. Therefore, for each positive integer $n$, there exist $c_{1}, \cdots, c_{n}$ in $P$ and not in any prime ideal in $\mathscr{P}^{*}$ such that no height one prime ideal in $R / Q$ contains more than one $c_{k}(k=1, \cdots, n)$, since each $\left(Q, c_{k}\right) R$ has only a finite number of minimal prime divisors. Hence, if $p_{k}$ is a minimal prime divisor of $\left(Q, c_{k}\right) R$ which is contained in $P$ and is such that height $P / p_{k}=$ height $P / Q-1$, then $E_{p_{k}}$ is Macaulay and $p_{k} \nsubseteq \cup N_{i}$. It follows that $Q=$ $\cap\left\{p ; p \in \mathscr{P}_{1}\right\}$.

To obtain another corollary to (2.2), the following lemma is needed.

Lemma 2.22. Let $\mathscr{S}$ be a set of prime ideals in a Noetherian ring $R$, let $I=\cap\{p ; p \in \mathscr{S}\}$, let $P$ be a minimal prime divisor of $I$, and let $c \in R, \notin P$. Then the following statements hold for $\mathscr{S}^{\prime}=$ $\{p \in \mathscr{S} ; c \notin p\}$ :

2.22.1. $P=\cap\{p ; p \in \mathscr{T}\}$, and $P R_{c}=\cap\left\{p R_{c} ; p \in \mathscr{T}\right\}$, where $\mathscr{T}=$ $\left\{p \in \mathscr{S}^{\prime} ; P \subseteq p\right\}$.

2.22.2. If $\mathscr{S}$ is big, then $\mathscr{S}^{\prime}$ is big.

2.22.3. If $\mathscr{S}$ is VS-open, then $\mathscr{S}^{\prime}$ is VS-open.

Proof. For (2.22.1) let $K=\cap\{p \in \mathscr{S} ; c \in p$ and $P \subseteq p\}$. Then $K \cap(\cap \mathscr{T})=P$, hence $P=\cap \mathscr{T}$, and (2.22.1) follows from this. The last two stements follow from the definitions and the fact that $R / p$ 
is Noetherian, for each $p \in \mathscr{S}$.

CoRollary 2.23. Let $R, E$, and $Q$ be as in (2.20), so $E_{Q}$ is Macaulay, and let $b \in R, \notin Q$. Then for each maximal ideal $M$ in $R$ such that $Q \subset M, Q=\cap\{p \in \mathscr{S}$; height $M / p=1$ and height $p=$ height $Q+$ height $M / Q-1\}$, where $\mathscr{S}=\{p \in S p e c R ; Q \subseteq p \subset M, b \notin p$, and $E_{p}$ is Macaulay\}. Moreover, if $R$ is local, then $\mathscr{S}$ is VS-open.

Proof. This is clear if height $M / Q=1$, and, if height $M / Q>1$, then the first statement follows from (2.19) and (2.22.1), and the last statement follows from (2.4.1) and (2.22.3).

REMARK 2.24. 2.24.1. In (2.23), if, moreover, $R$ is a Hilbert ring, then $Q=\cap\{M ; M$ is a maximal ideal in $R, Q \subset M, b \notin M$, and $R_{M}$ is Macaulay\} by (2.20) and (2.22.1).

2.24.2. If $R$ satisfies the first chain condition for prime ideals [8, p. 123], then it follows from (2.23) that $Q=\cap\{p \in \operatorname{Spec} R ; Q \subseteq p, b \notin p$, depth $p=1$, and $R_{p}$ is Macaulay\}. Also, depth $p=1$ if and only if height $p=$ altitude $R-1$.

2.24.3. It follows immediately from (2.23) that, if $P$ is a prime ideal in a Noetherian ring $R$ and $b \in P$ is such that $b$ is not in some minimal prime ideal $q \subset P$, then $q=\cap\{p \in \operatorname{Spec} R ; q \subseteq p \subset P, b \notin p$, height $P / p=1$, height $p=$ height $P / q-1$, and $R_{p}$ is Macaulay .

One final corollary, (2.25) below, which pertains to rather recent research in local ring theory will be given. The following background information on the corollary should be noted: It is known that, if $P$ is a prime ideal in an unmixed (resp., quasi-unmixed) local ring $R$, then $R_{P}$ is unmixed (resp., quasi-unmixed) [7, Proposition 6] (resp., [10, Lemma 2.5]). It was recently shown that there exist quasiunmixed local rings which are not unmixed (by [1, Proposition 3.3] together with [12, Proposition 3.5]). Thus it seems natural to inquire if there exist prime ideals $P$ of depth one in a quasi-unmixed local ring $R$ such that $R_{P}$ is unmixed. It follows from $(2.25)$ below that the answer is yes.

CoROLLARY 2.25. Let $R$ be a local ring such that altitude $R=$ $a>1$. Then $\mathscr{S}=\left\{p \in \operatorname{Spec} R ; R_{p}\right.$ is unmixed $\}$ is VS-open in Spec $R$ and $\{p \in \mathscr{S}$; height $p=a-1\}$ is an infinite set and is Zariski dense in Spec R.

Proof. Tne first statement follows from (2.4.1) with $E=R$, since a Macaulay local ring is unmixed [8, (34.9)]. The second statement follows from [8, (34.9)] and (2.19) with $Q$ a minimal prime ideal and $P$ the maximal ideal in $R$. 
This section will be closed with the following proposition and remarks. The proposition is closely related to a number of the results in this section, and it gives, in particular, some interesting supplementary information to (2.5.2) in the case $E=R$.

Proposition 2.26. Let $P_{1}, \cdots, P_{e}$ be prime ideals in a Noetherian ring $R$ which have the same height say height $P_{i}=a>0$. Then there exist $b_{1}, \cdots, b_{a-1}$ in $\cap P_{i}$ such that, with $B_{j}=\left(b_{1}, \cdots, b_{j}\right) R$ :

2.26.1. Height $B_{j}=j(j=0,1, \cdots, a-1)$.

2.26.2. If $Q \nsupseteq P_{i}(i=1, \cdots, e)$ is a prime ideal in $R$ such that $B_{j} \subseteq Q$, for some $j \geqq 1$, then the images of $b_{1}, \cdots, b_{j}$ in $R_{Q}$ are an $R_{Q}$-sequence.

2.26.3. If $P$ is a minimal prime divisor of $B_{k}$, for some $k(0 \leqq$ $k \leqq a-1)$, then $R_{P}$ is Macaulay and $B_{j} R_{P}$ is a primary ideal ( $j=$ $0,1, \cdots, k)$.

2.26.4. If $a>1$ and $\mathscr{P}_{i}$ is the infinite set of height $a-1$ prime ideals $p \subset P_{i}$ such that $B_{a-2} \subset p$ and $R_{p}$ is Macaulay, then, for each $i=1, \cdots, e$, at most a finite number of $p \in \mathscr{P}_{i}$ don't satisfy: $B_{j} R_{p}$ is primary, for $j=0,1, \cdots, a-2$.

Proof. The proposition is trivial for $a=1$, so it may be assumed that $a>1$. Let $\mathfrak{P}_{0}$ be the set of prime divisors $q$ of zero in $R$ such that $q \nsupseteq P_{i}(i=1, \cdots, e)$, let $\xi_{0}$ be the set of minimal prime divisors of zero in $R$, and let $\mathfrak{U}_{0}$ be the set of height one prime ideals in $R$ which contain more than one element in $\mathfrak{P}_{0}$. Then, $\mathfrak{U}_{0} \cup \mathfrak{P}_{0}$ is a finite set of prime ideals, by (2.1). Thus, since $a>1$, there exists $b_{1} \in \cap P_{i}$ such that $b_{1}$ isn't in any ideal in $\mathfrak{A}_{0} \cup \mathfrak{P}_{0}$. Then, with $B_{1}=b_{1} R$, it is readily checked that, if $a=2$, then (2.26.1)-(2.26.3) hold; and (2.26.4) holds by (2.1).

Assume $a>2$ and $\mathfrak{P}_{k-1}, \mathfrak{F}_{k-1}, \mathfrak{A}_{k-1}$, and $b_{1}, \cdots, b_{k}$ have been defined $(1 \leqq k<a-1)$, and let $\mathfrak{P}_{k}$ be the set of prime divisors $q$ of $B_{k}$ such that $q \nsupseteq P_{i}(i=1, \cdots, e)$. Assume (2.26.1) holds for $j=0,1 \cdots, k$, let $\mathfrak{F}_{k}$ be the set of minimal prime divisors of $B_{k}$, and let $\mathfrak{A}_{k}$ be the set of height $k+1$ prime ideals in $R$ which contain an element in $\mathfrak{F}_{k}$ and also contain more than $k+1$ prime ideals in $\bigcup_{0}^{k} \Re_{j}$. Assume further that $b_{1}, \cdots, b_{k}$ have been chosen such that (2.26.2) holds for $j \leqq k$ and, for $0 \leqq h \leqq k$ and each $p \in \widetilde{F}_{h}, B_{j} R_{p}$ is a primary ideal $(j=0,1, \cdots, h)$. Then $U=\left(\bigcup_{0}^{k} \mathfrak{\Re}_{j}\right) \cup \mathfrak{A}_{k}$ is a finite set of prime ideals, by (2.1). Therefore, there exists $b_{k+1}$ in $\cap P_{i}$ which is not in any prime ideal in $U$. Then it is easily checked that, if $P$ is a minimal prime divisor of $B_{k+1}=\left(B_{k}, b_{k+1}\right) R$, then $R_{P}$ is Macaulay and $B_{j} R_{P}$ is a primary ideal $(j=0,1, \cdots, k+1)$, and (2.26.1) and (2.26.2) hold for $j \leqq k+1$. Therefore, it follows that the desired elements $b_{1}, \cdots$, $b_{a-1}$ exist such that (2.26.1)-(2.26.3) hold. 
To show that (2.26.4) holds, note that, if $p \in \mathscr{P}_{i}$, then there exists a minimal prime divisor $q$ of $B_{a-2}$ contained in $p$, and then $B_{j} R_{q}$ is primary $(j=0,1, \cdots, a-2)$, by (2.26.3). Hence, since $B_{a-2}$ has only a finite number of minimal prime divisors contained in $P_{i},(2.26 .4)$ holds by (2.1).

REMARK 2.27. Let the notation be as in (2.26).

2.27.1. If the $P_{i}$ are maximal, then (2.26.2) shows that there exist $b_{1}, \cdots, b_{a-1}$ in $\cap P_{i}$ such that, for all prime ideals $P \notin\left\{P_{1}, \cdots\right.$, $\left.P_{e}\right\}$ such that $B_{j} \subseteq P$, for some $j$, the images in $R_{P}$ of $b_{1}, \cdots, b_{j}$ is an $R_{P}$-sequence.

2.27.2. It follows from (2.1) and (2.26.3) that, if $R$ is Hilbert and altitude $R=a$, then there are infinitely many maximal ideals $M$ in $R$ such that $R_{M}$ is Macaulay and $B_{j} R_{M}$ is primary $(j=0,1, \cdots$, $a-1)$.

2.27.3. If $P$ is a prime ideal in $R$ such that $a=$ height $P \geqq 2$, then (2.26.4) shows that there exist an infinite subset $\mathscr{P}^{\prime}$ of the set of prime ideals $Q \subset P$ such that height $Q=$ height $P-1$ and $R_{Q}$ is Macaulay such that $Q \in \mathscr{P}^{\prime}$ if and only if there exists a system of parameters $c_{1}, \cdots, c_{a-1}$ in $R_{Q}$ such that $\left(c_{1}, \cdots, c_{j}\right) R_{Q}$ is a primary ideal $(j=0,1, \cdots, a-1)$.

2.27.4. Let $Q_{1}, \cdots, Q_{z}$ be prime ideals in $R$ such that no $P_{i}(i=1$, $\cdots, e)$ is contained in $\bigcup Q_{j}$. Then the proof of $(2.26)$ can be readily adapted to show that the elements $b_{1}, \cdots, b_{a-1}$ can be chosen to satisfy the further condition that no $b_{h}$ is contained in $\cup Q_{j}$.

REMARK 2.28. It is natural to inquire if (2.5.2) holds on replacing Macaulay by Gorenstein (or, regular). The answer is no. For, let $R=A\left[X_{1}, \cdots, X_{n}\right]$, where $(A, q)$ is a primary ring whose zero ideal isn't irreducible, and where the $X_{i}$ are indeterminates. Then, for each prime ideal $P$ in $R, R_{P}$ isn't Gorenstein, since $q R \subseteq P$ and $R_{q R}$ isn't Gorenstein.

3. Condition $(*)$ and affine rings. In this section two theorems concerning a finitely generated ring $A$ over a Noetherian ring $R$ will be proved. The first, Theorem 3.9, shows, in particular, that if $A$ is an integral domain, then condition $(*)$ (Definition 3.2) is inherited by $A$, and the second, Theorem 3.12, shows that, if $R$ is a semi-local domain and altitude $R>1$, then "most" finitely generated integral domains over $R$ which aren't integrally dependent on $R$ satify condition $(*)$.

Definition 3.1. For a ring $R$, let $\mathscr{C}(R)$ be the set of maximal ideals $M$ in $R$ such that $R_{M}$ is Macaulay.

Definition 3.2. A ring $R$ is said to satisfy condition (*) in case 
$\operatorname{Rad} R=\cap\{M ; M \in \mathscr{C l}(R)\}$.

It follows from (2.17) that a Noetherian Hilbert ring satisfies condition (*). The next lemma shows that there exist rings which satisfy condition $\left(^{*}\right)$ and yet are neither locally Macaulay nor Hilbert rings (since $R[X]$ is Hilbert if and only if $R$ is Hilbert [5, Theorem $31])$.

LEMma 3.3. If $R$ is a Noetherian ring, then, for all $n \geqq 1$, the polynomial ring $R\left[X_{1}, \cdots, X_{n}\right]$ satisfies condition $\left(^{*}\right)$.

Proof. It may clearly be assumed that $n=1$. Let $P \in \mathscr{G}=$ $\left\{P ; P\right.$ is a $G$-ideal in $R$ and $R_{P}$ is Macaulay\}. ( $\mathscr{G}$ isn't empty by (2.12) with $E=R$.) If $P$ is a maximal ideal in $R$, then let $\mathscr{P}(P)$ be the set of maximal ideals in $R[X]$ which contain $P R[X]$. Then $R[X]_{N}$ is Macaulay, for each $N \in \mathscr{P}(P)$, and $P R[X]=\cap\{N ; N \in \mathscr{P}(P)\}$. On the other hand, if $P$ isn't maximal, then let $M_{1}, \cdots, M_{e}$ be the maximal ideals in $R$ which contain $P$. Let $b \in \cap M_{j}, \notin P$, and, for $i>0$, let $P_{i}=\left(P, b^{i} X-1\right) R[X]$. Then the $P_{i}$ are distinct prime ideals, each $R[X]_{P_{i}}$ is Macaulay, $P R[X]=\cap\left\{P_{i} ; i>0\right\}$, and each $P_{i}$ is a maximal ideal in $R[X]$. Hence, since $\operatorname{Rad} R=\cap\{P$; $P \in \mathscr{G}\}(2.12)$, the lemma follows from $\operatorname{Rad} R[X]=(\operatorname{Rad} R) \cdot R[X]=$ $\cap\{P R[X] ; P \in \mathscr{G}\}$.

COROLlary 3.4. If $I$ is an ideal in a Noetherian ring $R$ and $X$ is an indeterminate, then $\operatorname{Rad} I R[X]=\cap\{M ; I R[X] \subset M$ and $M / I R[X] \epsilon$ $\mathscr{L C}(R[X] / I R[X])\}$. In particular, if $b$ is a nonunit regular element in $R$, then $\operatorname{Rad} b R[X]=\cap\{M ; b \in M \in \mathscr{C}(R[X])\}$.

Proof. This follows from $R[X] / I R[X]=(R / I)[X]$ and [5, Theorem $156]$.

On the other hand, of course, there are height one prime ideals in $R[X]$ which aren't even an intersection of maximal ideals; for example, $X R[X]$ when $R$ is a local domain.

To prove the first theorem in this section, a number of lemmas will first be proved.

Lemma 3.5. Let $R$ be a Noetherian ring.

3.5.1. If $A$ is a Noetherian ring such that $A$ is integrally dependent on $R$ and $R \subseteq A \subseteq R_{c}$, for some nonzero-divisor $c \in R$, and if $A$ satisfies condition $\left({ }^{*}\right)$, then $R$ does.

3.5.2. If $R$ satisfies condition (*), then, for all nonzero-divisors $c$ in $R$ and for each ring $A$ such that $R \subseteq A \subseteq R_{c}, A$ satisfies condition $(*)$.

3.5.3. If $R$ satisfies condition $\left({ }^{*}\right)$, then each free principal integral 
extension ring of $R$ (that is, $R[X] /(f)$, where $f$ is monic) satisfies condition $(*)$.

Proof. 3.5.1. If $A$ satisfies condition $\left({ }^{*}\right)$, then $A_{c}=R_{c}$ does and $\cap\left\{M A_{c} ; M \in \mathscr{C}(A)\right\}=(0)$, by (2.22.1). Hence, since $A$ is integrally dependent on $R, R$ satisfies condition $(*)$. For (3.5.2), if $R$ satisfies condition (*), then $\cap\left\{M R_{c} ; M \in \mathscr{C}(R)\right\}=(0)$, by (2.22.1), so $\cap\left\{M R_{c} \cap\right.$ $A ; M \in \mathscr{C}(R)\}=(0)$. Hence, since $R_{c} / M R_{c}=R / M$ and $R_{M}=\left(R_{c}\right)_{M R_{c}}$ (if $c \notin M), A$ satisfies condition $\left(^{*}\right)$.

For (3.5.3), let $B=R[X] /(f)$. Then, for each maximal ideal $M$ in $R,(\operatorname{Rad} M B)^{d} \subseteq M B$, where $d$ is the degree of $f$ (consider $R[X] /$ $(M, f))$. Therefore, if $u \in \cap\{N ; N \in \mathscr{M}(B)\}$, then, for each $M \in \mathscr{C}(R)$, $u \in \operatorname{Rad} M B$ (by freeness), so $u^{d} \in \cap\{M B ; M \in \mathscr{M}(R)\}=$ (by freeness) $(\cap\{M ; M \in \mathscr{C}(R)\}) B$, and so $u^{d}$ is nilpotent. Thus $u \in \operatorname{Rad} B$, hence $B$ satisfies condition $(*)$.

In (3.6), we shall utilize [3, (6.10.6)]. The result is essentially local, and passing from the language of preschemes to the language of commutative rings we find that it asserts the following:

Let $R$ be a Noetherian ring, let $E$ be a finite $R$-module, and let $I$ be an ideal in $R$ such that $q=\operatorname{Rad} I$ is prime and $E_{q} \neq(0)$. Then there exists $r \in R, \notin q$ such that, for each prime ideal $Q$ in $R$ such that $q \subseteq Q$ and $r \notin Q$, the following holds:

$\operatorname{Dim} E_{Q}=\operatorname{Dim} E_{q}+\operatorname{Dim}(R / I)_{Q}$ and Prof $E_{Q}=\operatorname{Prof} E_{q}+\operatorname{Prof}(R / I)_{Q / I} \cdot$

Lemma 3.6. Let $q$ be a minimal prime ideal in a Noetherian ring $R$. Then there exists an element $r \in R, \notin q$ such that, for each prime ideal $Q$ in $R$ which contains $q$ but not $r, R_{Q}$ is Macaulay if and only if $(R / q)_{Q / q}$ is Macaulay.

Proof. If $s \in R, \notin q$, then it clearly suffices to prove the lemma for $R_{s}$ instead of $R$. Hence it may be assumed that $q$ is nilpotent. Then, with $q=I$ and $R=E$ in Grothendieck's result quoted above, $\operatorname{Dim} E_{q}=$ Prof $E_{q}=0$ (since $q$ is nilpotent). Therefore, for each prime ideal $Q$ in $R$ such that $r \notin Q$, altitude $R_{Q}=$ altitude $(R / q)_{Q / q}$ and Prof $R_{Q}=\operatorname{Prof}(R / q)_{Q / q}$. Clearly, then, for each such prime ideal $Q$ in $R, R_{Q}$ is Macaulay if and only if $(R / Q)_{Q / q}$ is Macaulay.

LEMMA 3.7. A Noetherian ring $R$ satisfies condition $\left(^{*}\right)$ if and only if, for each minimal prime ideal $q$ in $R, R / q$ satisfies condition $(*)$.

Proof. Let $R$ satisfy condition $\left(^{*}\right)$ and let $q$ be a minimal prime 
ideal in $R$. Choose $r \in R$ as in (3.6). Since $q=\cap\{M ; q \subseteq M \in \mathscr{L}(R)\}$, by hypothesis, $q=\cap\{M ; q \leqq M \in \mathscr{L}(R)$ and $r \notin M\}$ (2.22.1). Hence, by (3.6), $R / q$ satisfies condition (*).

Conversely, let $q_{1}, \cdots, q_{g}$ be the minimal prime ideals in $R$, and assume that each $R / q_{i}$ satisfies condition $\left({ }^{*}\right)$. Then, for each $i=1, \cdots, g$ and with $r_{i}$ as in (3.6), $q_{i}=\cap\left\{M ; q \subseteq M, M / q_{i} \in \mathscr{M}\left(R / q_{i}\right)\right.$, and $\left.r_{i} \notin M\right\}$, by (2.22.1). Thus, by (3.6), $q_{i}=\cap\left\{M ; q_{i} \subseteq M \in \mathscr{M}(R)\right.$ and $\left.r_{i} \notin M\right\}(i=$ $1, \cdots, g)$, hence $R$ satisfies condition $\left(^{*}\right)$.

Corollary 3.8. A Noetherian ring $R$ satisfies condition $\left({ }^{*}\right)$ if and only if $R /(\operatorname{Rad} R)$ satisfies condition $(*)$.

Proof. Clear by (3.7).

Since a finitely generated ring over a Noetherian Hilbert ring is again such a ring, it follows from (2.17) that a finitely generated ring over a Noetherian Hilbert ring satisfies condition $(*)$. The following theorem can be considered a generalization of this result.

THeOREM 3.9. Let $A$ be a finitely generated extension ring of a Noetherian ring $R$, and assume $R$ satisfies condition (*). Then $A$ satisfies condition $\left(^{*}\right)$ if, for each minimal prime ideal $Q$ in $A$, at least one of the following conditions holds:

3.9.1. $Q \cap R$ is minimal.

3.9.2. $R /(Q \cap R)$ satisfies condition $(*)$.

3.9.3. $A / Q$ is not algebraic over $R /(Q \cap R)$.

Proof. $A / Q$ satisfies condition (*) if (3.9.3) holds, by (3.3). Also (3.9.1) implies (3.9.2), by (3.7). Therefore, since $A$ satisfies condition $\left.{ }^{*}\right)$ if each $A / Q$ does (3.7), it suffices to prove: If $A$ is a finitely generated integral domain over $R$ and $R$ satisfies condition $(*)$, then $A$ satisfies condition $\left({ }^{*}\right)$.

For this, there exist algebraically independent elements $X_{1}, \cdots$, $X_{n}$ in $A$ over $R$, and elements $a_{1}, \cdots, a_{k}$ in $A$ integral over $R_{n}=$ $R\left[X_{1}, \cdots, X_{n}\right]$ such that $B=R_{n}\left[a_{1}, \cdots, a_{k}\right] \subseteq A \subseteq B[1 / b]$, for some nonzero element $b \in B$. Therefore, by (3.5.2), it suffices to prove $B$ satisfies condition $\left(^{*}\right)$. By (3.3), $R_{n}$ satisfies condition $\left(^{*}\right)$, so it may be assumed that $B=R\left[a_{1}, \cdots, a_{k}\right]$. Then the coefficients of the minimal polynomial of $a_{1}$ over the quotient field of $R$ are in a finite integral extension $R_{1}$ of $R$ contained in the quotient field of $R$, and $R_{1}\left[a_{1}\right]$ is a free principal integral extension domain of $R_{1}$. Therefore, by (3.5.2) and (3.5.3), $R_{1}\left[a_{1}\right]$ satisfies condition $(*)$, hence $R\left[a_{1}\right]$ does, by (3.5.1). Therefore, the theorem follows by induction on $k$. 
Corollary 3.10. Let $R$ and $A$ be as in (3.9). If $A$ contains an indeterminate over $R$, say $t$, such that, for each minimal prime ideal $Q$ in $A, Q \cap R[t]=(Q \cap R) R[t]$, then $A$ satisfies condition $\left(^{*}\right)$.

Proof. This follows from (3.3) and (3.9), since $Q \cap R[t]=(Q \cap$ $R) R[t]$ implies $\operatorname{trd}(A / Q) /(R /(Q \cap R))>0$.

Condition (b) in (3.9) suggests a way to construct extensions for which $R$ satisfies condition (*) and $A$ does not. In fact, let $R$ be a Noetherian ring which satisfies condition $\left(^{*}\right)$ and which has a prime ideal $P$ such that $R / P$ doesn't satisfy condition $\left(^{*}\right)$. (For example, let $R_{0}$ be a local domain such that altitude $R_{0}=1$, let $R=R_{0}[X]$, where $X$ is an indeterminate, and let $P=X R$.) Let $A=(R / P) \oplus R$, and let $f: R \rightarrow A$ by $f(r)=(r+P, r)$. Then $A$ is finitely generated over $f(R)$ by $(1+P, 0), f(R)$ satisfies condition $\left(^{*}\right)$ (since $f$ is a monomorphism), but $A$ doesn't satisfy condition (*), by (3.7), since $Q=(0,1) A$ is a minimal prime ideal in $A$ such that $A / Q=R / P$ doesn't satisfy condition $(*)$.

The following lemma is needed to shorten the proof of (3.12) below.

LEMMA 3.11. Let $b$ and $c$ be non-unit regular elements in $a$ Noetherian ring $R$, let $y=c / b$, let $I=(y, b) R[y]$, and assume $b \notin$ Rad cR. If $I \neq R[y]$, then height $I=2$.

Proof. If $1 \notin I$, then height $I \leqq 2$. Suppose $Q$ is a height one prime ideal in $R[y]$ such that $I \subseteq Q$. Localizing at $Q \cap R$, it may be assumed that $R$ is a local ring with maximal ideal $M$ and $Q \cap R=M$. Since height $Q=1$, there exist $s \in R[y], \notin Q$, and $n>0$ such that $s b^{n} \in y R[y]$. Therefore, with $s=\Sigma r_{i} y^{i}\left(r_{i} \in R\right), r_{0} b^{n} \in y R[y]$. Now $r_{0} \notin M$, since $s \notin Q$, so $r_{0}$ is a unit in $R$ and $b^{n} \in y R[y]$. But multiplying by a suitable power of $b$ will clear of fractions on the right and will show that $b \in \operatorname{Rad} c R$, a contradiction. Thus height $I=2$.

Following the proof of the next theorem, an example will be given to show that the assumption that altitude $R>1$ is necessary. Before stating the theorem, it should be noted that there may exist height one maximal ideals in the integral closure of a Noetherian domain $R$. even if $R$ is local and altitude $R>1$; for example, see [8, Example 2, pp. 203-205].

THEOREM 3.12. Let $A$ be a finitely generated integral domain 
over a semi-local domain $R$, and let altitude $R>1 . \quad A$ satisfies condition (*) if and only if $A \nsubseteq R_{c}^{\prime}$, where $R^{\prime}$ is the integral closure of $R$ in the quotient field of $A$ and $c$ is either a unit in $R^{\prime}$ or depth $c R^{\prime}=0$.

Proof. If $\operatorname{trd} A / R>0$, then $A$ satisfies condition (*), by (3.9), and $A$ isn't contained in any quotient ring of $R^{\prime}$, so it may be assumed that $A$ is algebraic over $R$. Then, since there is a finite integral extension ring $B$ of $R$ contained in $A$ such that $B$ and $A$ have the same quotient field, it may be assumed that $R$ and $B$ have the same quotient field.

Assume first that $A \subseteq R_{c}^{\prime}$, for some such element $c \in R^{\prime}$. Then, since $A$ is finitely generated over $R$, there exist $c_{1}, \cdots, c_{k}$ in $R^{\prime}$ such that $B=R\left[c, c_{1}, \cdots, c_{k}\right] \subseteq A[c] \leqq B_{c}$. Since $B_{c}$ has only a finite number of maximal ideals (since its integral closure $R_{c}^{\prime}$ does (every prime ideal in $B$ which contains $c$ has height one, since depth $\left.c R^{\prime}=0\right)$ ), $B_{c}$ doesn't satisfy condition $\left({ }^{*}\right)$. Therefore, since $(A[c])_{c}=B_{c}$, it follows from (3.5.2) that $A[c]$ doesn't, hence $A$ doesn't satisfy condition (*), by (3.9).

Conversely, assume, for each such element $c \in R^{\prime}, A \nsubseteq R_{c}^{\prime}$. Then, there exists $x \in A$ such that $x \notin R_{M^{\prime}}^{\prime}$, for some maximal ideal $M^{\prime}$ in $R^{\prime}$ such that height $M^{\prime}>1$. Fix one such $M^{\prime}$. By (3.5.1), by adjoining to $R$ a finite number of elements from $R^{\prime}$, it may be assumed that $R$ and $R^{\prime}$ have the same number of maximal ideals, so, in particular, $R_{M^{\prime}}^{\prime}$ is the integral closure of $R_{M}$, where $M=M^{\prime} \cap R$. If $1 / x \in R_{M^{\prime}}^{\prime}$, then $1 / x \in M^{\prime} R_{M^{\prime}}^{\prime} \cap R_{M}[1 / x]$, so $R_{M}[x]=R_{M}[1 / x, x]$ is a Noetherian Hilbert domain $[4,(10.5 .8)]$. Let $\mathscr{P}=\{P \in \operatorname{Spec} R ; P \subset M, R / P$ is local, depth $P=1$, and $R_{P}$ is Macaulay , let $x=b / c$ with $b$ and $c$ in $M$, and let $\mathscr{S}=\left\{N \in \mathscr{C}\left(R_{M}[x]\right) ; N=P\left(R_{M}\right)_{c} \cap R_{M}[x]\right.$, for some $\left.P \in \mathscr{P}\right\}$. (For each $P \in \mathscr{P}, P\left(R_{M}\right)_{c} \cap R_{M}[x]$ is maximal, since each $P\left(R_{M}\right)_{c}$ is maximal and $R_{M}[x]$ is Hilbert.) Then $\cap\{P ; P \in \mathscr{P}\}=(0)$ (by 2.21) with $Q=(0)$ and $N_{1}, \cdots, N_{g}$ the other maximal ideals in $R$ ), so $\cap\{N ; N \in \mathscr{S}\}=(0)$, by (2.22.1). Fix $N \in \mathscr{S}$, let $p=N \cap R[x]$, and let $Q$ be a maximal ideal in $R[x]$ such that $p \subseteq Q$. Then $Q \cap R \subseteq M$, since $p \cap R \in \mathscr{P}$. Therefore, since $N$ is maximal, it follows that $Q=p$ (since $M^{\prime}$ is lost in $R^{\prime}[x]$ implies $M$ is lost in $\left.R[x]\right)$. Hence, since $\cap\{p ; p=N \cap R[x]$, for some $N \in \mathscr{S}\}=(0), R[x]$ satisfies condition $\left(^{*}\right)$. Therefore, $A$ satisfies condition $(*)$, by (3.9).

Therefore, assume $x$ and $1 / x \notin R_{M^{\prime}}^{\prime}$, and let $x=b / c$ with $b$ and $c \in M^{\prime}$. Then $b R^{\prime}: c R^{\prime}$ and $c R^{\prime}: b R^{\prime}$ have no common prime divisors, since, for each height one prime ideal $p$ in $R^{\prime}, b / c$ or $c / b \in R_{p}^{\prime}$. Let $d \in b R^{\prime}: c R^{\prime}$ such that $d$ isn't in any prime divisor of $c R^{\prime}: b R^{\prime}$, and let $e \in c R^{\prime}: b R^{\prime}$ such that $d c=b e$. Then $x=d / e$, so $(d, e) R^{\prime} \leqq M^{\prime}$ (since $M^{\prime} R^{\prime}[x]$ is a depth one prime ideal [17, Corollary, p. 20]), so it 
may be assumed that $b=d$ and $c=e$ and, by (3.5.1), that $b$ and $c$ are in $R$. Then $b \notin \operatorname{Rad} c R$, since, for each prime divisor $p$ of $c R^{\prime}: b R^{\prime}$, $p$ is a prime divisor of $c R^{\prime}$, hence $p \cap R$ is a prime divisor of $c R$ [8, (33.11)] and $b \in p \cap R$. Let $y=1 / x=c / b$, and fix $j>0$. Then $b c$ is not in any minimal prime divisor of $\left(y-b^{j}\right) R[y]$; for, if $Q$ is a height one prime ideal in $R$ such that $\left(b c, y-b^{j}\right) R[y] \subseteq Q$, then $b$ or $c$ and $y-b^{j}$ are in $Q$, and this implies the contradiction $(b, y) R[y] \subseteq Q$ (3.11). Thus, for all $j \geqq 1$, there exists a minimal prime divisor $p_{j}$ of $\left(c-b^{j+1}\right) R$ contained in $M$ such that $b c \notin p_{j}$. Fix $j$, and let $\mathscr{P}_{j}=$ $\left\{P \in \mathscr{P} ; b c \notin P\right.$ and $\left.p_{j} \subseteq P\right\}$, where $\mathscr{P}$ is as in the preceding paragraph. Then it follows from (2.21) and (2.23) (with $P=M, Q=p_{j}$, and $N_{1}$, $\cdots, N_{g}$ the other maximal ideals in $R$ ) that $p_{j}=\cap\left\{P ; P \in \mathscr{P}_{j}\right\}$, so $p_{j} R_{c} \cap R[x]=\cap\left\{N ; N \in \mathscr{S}^{\prime}\right\}$, where $\mathscr{S}^{\prime}=\left\{N ; N=P R_{c} \cap R[x]\right.$, for some $\left.P \in \mathscr{P}_{j}\right\}$ (2.22.1). Now, if $N \in \mathscr{S}^{\prime}$ then $N$ is a maximal ideal in $R[x]$ (since $b^{j} x-1 \in N$ and $R / P \subseteq R[x] / N \subseteq R_{c} / P R_{c}=$ the quotient field of $R / P)$. Since $\cap\left\{p_{j} R_{c} \cap R[x] ; j \geqq 1\right\}=(0), R[x]$ satisfies condition (*). Hence $A$ satisfies condition (*), by (3.9).

A necessary and sufficient condition for $A$ to satisfy condition (*) was just given in (3.12), assuming $a=$ altitude $R>1$. If $a=1$, then the condition isn't necessary. For, let $R$ be a discrete valuation ring whose maximal ideal is generated by $c$, and let $A=R_{c}$. Then $A$ is finitely generated over $R, A \subseteq R_{c}=R_{c}^{\prime}$, and depth $c R^{\prime}=0$, but $A$ satisfies condition (*), since $A$ is a field. On the other hand, the condition is sufficient when $a=1$. For, if $A \nsubseteq R_{c}^{\prime}$, for all nonzero $c \in R^{\prime}$, then $A$ isn't contained in the quotient field of $R^{\prime}$ (since $a=1$ and $R^{\prime}$ is quasi-semi-local), so $A$ is transcendental over $R$. Therefore $A$ is finitely generated over a Noetherian ring of altitude greater than one, hence $A$ satisfies condition (*), by (3.12).

Corollary 3.13. Let $R$ be a semi-local domain such that altitude $R>1$, let $S$ be the integral closure of $R$ in its quotient field, and let $A$ be a finitely generated integral domain over $R$ such that $A$ isn't integral over $R$. Then A satisfies condition $\left(^{*}\right)$ in each of the following cases:

3.13.1. $R=S$.

3.13.2. $S$ is quasi-local.

3.13.3. S has no height one maximal ideals.

3.13.4. $R$ satisfies the second chain condition for prime ideals.

Proof. (3.13.1) follows from (3.12), since the integral closure $R^{\prime}$ of $R$ in the quotient field of $A$ has no height one maximal ideals [8, (10.14)]. Clearly, (3.13.2) implies (3.13.3), and (3.13.4) implies (3.13.3) 
[12, Theorem 3.1 and Proposition 3.5]. Finally, (3.13.3) implies that $R^{\prime}$ has no height one maximal ideals [8, (10.14)], so (3.13.3) follows from (3.12).

4. Rees rings and $R$-sequences. Let $B=\left(b_{1}, \cdots, b_{w}\right) R$ be an ideal in a semi-local ring $\left(R ; M_{1}, \cdots, M_{e}\right)$ such that $B \cong J=\cap M_{i}$, let $t$ be an indeterminate, and let $u=1 / t$. The Rees ring $\mathscr{R}=\mathscr{R}(R, B)$ of $R$ with respect to $B$ is defined to be the subring $\mathscr{R}=R\left[t b_{1}, \cdots\right.$, $\left.t b_{w}, u\right]$ of $R[t, u]$.

The following remark summarizes the basic facts on Rees rings which will be used in what follows.

REMARK 4.1. The elements in $\mathscr{R}$ are finite sums $\sum_{-m}^{n} c_{i} t^{i}$, where $c_{i} \in B^{i}$ (with the convention that $B^{i}=R$, if $i \leqq 0$ ). Thus, $\mathscr{R}$ is a graded Noetherian ring, $u$ isn't a divisor of zero in $\mathscr{R}$, and $u^{i} \mathscr{R} \cap$ $R=B^{i}$, for all $i \geqq 0$. For a homogeneous ideal $H$ in $\mathscr{R}$ and $-\infty<$ $n<\infty$, let $[H]_{n}$ denote the set of elements $r \in R$ such that $r t^{n} \in H$. Then, if also $K$ is a homogeneous ideal in $\mathscr{R}$, then $[H+K]_{n}=[H]_{n}+$ $[K]_{n}$, and $H \subseteq K$ if and only if $[H]_{n} \subseteq[K]_{n}$, for all n. Also, $B^{n} \supseteqq$ $[H]_{n} \supseteqq[H]_{n+1} \supseteqq B[H]_{n}$, for all $n[16$, p. 11]. In particular, for an ideal $C$ in $R$, let $C^{*}=C R[t, u] \cap \mathscr{R}$, so $C^{*}$ and $\left(C^{*}, u\right) \mathscr{R}$ are homogeneous ideals in $\mathscr{R}$, and $\left[\left(C^{*}, u\right) \mathscr{R}\right]_{n}=\left(C \cap B^{n}\right)+B^{n+1}$. It follows that $B^{*}=\left(b_{1} t, \cdots, b_{w} t\right) \mathscr{R}$, and $\mathscr{C l}_{i}=\left(M_{i}^{*} u\right) \mathscr{R}=\left(M_{i}, u, B^{*}\right) \mathscr{R}$ are the maximal homogeneous ideals in $\mathscr{R}(i=1, \cdots, e)$. Also, it follows easily from the definition that, if $C=\cap Q_{j}$ is a normal decomposition of $C$, where $Q_{j}$ is $P_{j}$-primary, then $P_{j}^{*}$ is prime, $Q_{j}^{*}$ is $P_{j}^{*}$-primary, and $C^{*}=\cap Q_{j}^{*}$ is a normal decomposition of $C^{*}$ [15, Theorem 1.5]. Further, height $C^{*}=$ height $C$, height $\mathscr{C}_{i}=$ height $M_{i}+1$, and altitude $\mathscr{R}=$ altitude $R+1$ [13, Remark 3.7].

Most of the results in this section follow from the following basic lemma.

Lemma 4.2. Let $\left(R ; M_{1}, \cdots, M_{e}\right)$ be a semi-local ring such that height $M_{i}=$ altitude $R=a(i=1, \cdots, e)$. Let $B$ be an ideal in $R$ such that $\operatorname{Rad} B=J=\cap M_{i}$, and let $\mathscr{R}=\mathscr{R}(R, B)$ be the Rees ring of $R$ with respect to $B$. Assume each $\mathscr{R}\left({ }_{\left(M_{i}^{*}, u\right)}\right)$ is Macaulay, and let $P_{1}, \cdots, P_{s}$ be homogeneneous prime ideals in $\mathscr{R}$. Assume height $P_{v}=k(v=1, \cdots, s)$ and either $u \in \cap P_{v}$ or $u \notin \cup P_{v}$. Then there exist homogeneous elements $x_{1}, \cdots, x_{j}$ in $\cap P_{v}$ such that every permutation of $x_{1}, \cdots, x_{j}, u$ is an $\mathscr{R}$-sequence and $j=k$ (if $u \notin \cup P_{v}$ ) or $j=k-$ 1 (if $u \in \cap P_{v}$ ).

Proof. Every prime divisor of each homogeneous ideal in $\mathscr{R}$ is homogeneous, hence is contained in $\mathscr{H}_{i}=\left(M_{i}^{*}, u\right) \mathscr{R}$, for some $i=1$, 
$\cdots, e$, and $\mathscr{R}_{S}$ is a Macaulay semi-local ring, where $S=\mathscr{R} \sim \cup \mathbb{C}_{i}$. Therefore, if $x_{1}, \cdots, x_{j}, u$ is an $\mathscr{R}$-sequence of homogeneous elements contained in $\left(J^{*}, u\right) \mathscr{R}$, then every permutation of it is an $\mathscr{R}$-sequence. Thus it suffices to prove the existence of homogeneous elements $x_{1}$, $\cdots, x_{j}$ in $\cap P_{v} \cap J^{*}$ such that some permutation of $x_{1}, \cdots, x_{j}, u$ is an $\mathscr{R}$-sequence and $j=k$ (if $u \notin \cup P_{v}$ ) or $j=k-1$ (if $u \in \cap P_{v}$ ).

Since this is clear if $k=0$, let $k>0$, and assume the lemma holds for such finite sets of homogeneous prime ideals in $\mathscr{R}$ of height less than $k$. For $v=1, \cdots, s$, let $Q_{v}$ be a homogeneous prime ideal in $\mathscr{R}$ such that $Q_{v} \subset P_{v}$, height $Q_{v}=k-1$, and either $u \in \cap Q_{v}$ or $u \notin \cup Q_{v}$. By induction, let $x_{1}, \cdots, x_{m}$ be homogeneous elements in $\cap Q_{v} \cap J^{*}$ such that $x_{1}, \cdots, x_{m}, u$ is an $\mathscr{R}$-sequence and $m=$ height $Q_{v}$ (if $u \notin \cup Q_{v}$ ) or $m+1=$ height $Q_{v}$ (if $u \in \cap Q_{v}$ ). Let $p_{1}, \cdots, p_{f}$ be the prime divisors of $\left(x_{1}, \cdots, x_{m}, u\right) \mathscr{R}$, let $\mathscr{P}=\left\{p_{1}, \cdots, p_{f}\right\}$, and fix $p \in \mathscr{P}$. Then: $(u, J) \mathscr{R} \cong p$, since $u \in p$ and $\operatorname{Rad} B=J$ imply $P \cap R=$ $M_{i}$, for some $i=1, \cdots, e$; and, $p$ is homogeneous, so $p \leqq \mathbb{L}_{i}$, for some $i=1, \cdots, e$. Therefore, since each $\mathscr{R}_{\mathbb{N}_{i}}$ is Macaulay, height $p=$ $m+1$. If one the $P_{v}$ is in $\mathscr{P}$, then $u \in P_{v}$ (hence $u \in \cap P_{v}$ ) and $u \notin$ $Q_{v}$ (hence $u \notin \cup Q_{v}$ ), and so every $P_{v}$ is in $\mathscr{P}$, hence the lemma holds. Thus it may be assumed that no $P_{v}$ is $\mathscr{P}$. Therefore, no $\mathscr{A}_{i}$ is in $\mathscr{P}$; for, if one $\mathscr{C l}_{i} \in \mathscr{P}$, then $m=a$ and so $\mathscr{S} \cong\left\{\mathscr{C}_{1}, \cdots, \mathscr{C}_{e}\right\}$, hence, since $k \geqq m+1$, it follows that all $P_{v} \in \mathscr{P}$; contradiction.

For $h=1, \cdots, f$, let $I_{h}=\bigcap_{j \neq h} p_{j} \cap B^{*} \cap P_{1} \cap \cdots \cap P_{s}$. Then $I_{h}$ is homogeneous, and $I_{h} \nsubseteq p_{h}$; for, $I_{h} \subseteq p_{h}$ implies $B^{*} \subseteq p_{h}$, hence $(u$, $\left.J, B^{*}\right) \mathscr{R} \subseteq p_{h}$, and so $p_{h}=\mathscr{C}_{i}$, for some $i=1, \cdots, e$; contradiction. Therefore, there exists a homogeneous element $z_{h} \in I_{h}$, $\notin p_{h}$; say $z_{h}=$ $r_{h} t^{t h}\left(d_{h}>0\right.$, since, for $\left.n \leqq 0,\left[I_{h}\right]_{n} \subseteq\left[B^{*}\right]_{n}=B \cong J \subseteq\left[p_{h}\right]_{n}\right)$. Thus, with $D_{h}=\pi_{j \neq h} d_{j}, x_{m+1}=\sum z_{h}^{D h}$ is a homogeneous element in $\cap P_{v} \cap J^{*}$ and not in $p_{1}, \cdots, p_{f}$ hence $x_{1}, \cdots, x_{m}, u, x_{m+1}$ is an $\mathscr{R}$-sequence.

REMARK 4.3. The homogeneous elements $x_{h}(h=1, \cdots, j)$ in $(4.2)$ must have positive degree, since $u, x_{k}$ is an $\mathscr{R}$-sequence and every homogeneous nonunit of nonpositive degree is in some prime divisor of $u . \mathscr{R}($ since $(\operatorname{Rad} u \mathscr{R}) \cap R=\operatorname{Rad}(u \mathscr{R} \cap R)=\operatorname{Rad} B=J)$.

The following two definitions are needed for (4.4) below.

A set of elements $y_{1}, \cdots, y_{a}$ in the Jacobson radical $J$ of a semilocal ring $R$ is a system of parameters in $R$ in case $\operatorname{Rad}\left(y_{1}, \cdots, y_{a}\right) R=$ $J$ and $a=$ altitude $R$ [8, p. 77].

If $B$ is an ideal in a ring $R$, and $x \in R$, then the degree of $x$ with respect to $B$, denoted $d_{B}(x)$, is the largest integer $n$ such that $x \in B^{n}$, if such $n$ exists. If $x \in B^{n}$, for all $n$, then $d_{B}(x)=\infty$.

Theorem 4.4. Let $\left(R ; M_{1}, \cdots, M_{e}\right), J, B, a$, and $\mathscr{R}$ be as in (4.2). Then $\mathscr{R}$ is locally Macaulay if and only if $R$ is Macaulay and there 
exists a system of parameters $y_{1}, \cdots, y_{a}$ in $R$ such that, for each $j=$ $1, \cdots$, a and for all $n \geqq 1,\left(y_{1}, \cdots, y_{j}\right) R \cap B^{n}=\sum_{1}^{j} y_{i} B^{n-d_{i}}$, where $d_{i}=$ $d_{B}\left(y_{i}\right)(i=1, \cdots, a)$.

Proof. If $a=0$, then altitude $\mathscr{R}=1$. Therefore $\mathscr{R}$ is locally Macaulay (since the prime divisors of $(0)$ in $\mathscr{R}$ are the ideal $q^{*}$ with $q$ a prime ideal in $R$, and so have height 0 ). Hence the theorem is trivially true upon defining $\sum_{1}^{a} y_{i} B^{n-d_{i}}$ to be zero when are no $y_{i}$. Therefore, let $a>0$.

If $\mathscr{R}$ is locally Macaulay, then $R$ is Macaulay [11, Theorem 3.8]. Also, by (4.2), there exist homogeneous elements $x_{1}, \cdots, x_{a} \in J^{*}$ such that $x_{1}, \cdots, x_{a}, u$ is an $\mathscr{R}$-sequence. For $j=1, \cdots, a$, let $X_{j}=\left(x_{1}\right.$, $\left.\cdots, x_{j}\right) \mathscr{R}$, let $x_{i}=y_{i} t^{d_{i}}$ with $y_{i} \in B^{d_{i}}$, and let $Y_{j}=\left(y_{1}, \cdots, y_{j}\right) R$. Then, since $x_{1}, \cdots, x_{j}, u$ is an $\mathscr{R}$-sequence $(j=1, \cdots, a), d_{B}\left(y_{i}\right)=d_{i}$. Also, $Y_{j}^{*}=Y_{j} R[t, u] \cap \mathscr{R}=X_{j}\left(\right.$ hence $\left.\operatorname{Rad} Y_{a}=J\right)$, since $R[t, u]=\mathscr{R}[1 / u]$ implies $Y_{j}^{*}=X_{j}: u^{k} \mathscr{R}$, for all large $k$. Hence $Y_{j} \cap B^{n}=\left[Y_{j}^{*}\right]_{n}=\left[X_{j}\right]_{n}=$ $\sum_{1}^{j} y_{i} B^{n-d_{i}}$, for $j=1, \cdots, a$ and for all $n$.

Conversely, assume $R$ is Macaulay and such a system of parameters $y_{1}, \cdots, y_{a}$ exists in $R$, let $Y_{j}=\left(y_{1}, \cdots, y_{j}\right) R(j=1, \cdots, a)$, and let $x_{i}=y_{i} t^{d_{i}}$. To prove $\mathscr{R}$ is locally Macaulay, let $N$ be a maximal ideal in $\mathscr{R}$.

(i) If $N=\mathscr{C}_{i}=\left(M_{i}^{*}, u\right) \mathscr{R}$, for some $i=1, \cdots, e$, then $\mathscr{L}_{i}$ is a minimal prime divisor of $\left(Y_{a}^{*}, u\right) \mathscr{R}$. Also, $Y_{j}^{*}=\left(x_{1}, \cdots, x_{j}\right) \mathscr{R}$, since $\left[Y_{j}^{*}\right]_{n}=\left[\left(x_{1}, \cdots, x_{j}\right) \mathscr{R}\right]_{n}$, for all $n$ (for $n \geqq 1$, by hypothesis; for $n<$ 1 , this is clear). Hence, since the prime divisors of the $Y_{j}^{*}$ are the ideals $P^{*}$ with $P$ a prime divisor of $Y_{j}$, and since $u \notin P^{*}, x_{1}, \cdots, x_{a}$, $u$ is an $\mathscr{R}$-sequence, and so $\mathscr{R}_{N}$ is Macaulay.

(ii) If $N \neq \mathscr{M}_{i}(i=1, \cdots, e)$, then either $u \notin N$, or $t b \notin N$, for some nonzero-divisor $b \in B$. (If $\left(u, B^{*}\right) \mathscr{R} \subseteq N$, then, since $\operatorname{Rad} B=$ $J, N=\mathscr{C}_{i}$, for some $i$; and, since $a>0$ and $R$ is Macaulay, $B$ can be generated by nonzero divisors [9, Lemma 10, p. 229].) If $u \notin N$, then $\mathscr{R}_{N}$ is Macaulay, since it is a quotient ring of $\mathscr{R}[1 / u]=R[t, u]$, and $R[t, u]$ is locally Macaulay, since $R$ is Macaulay. If $t b \notin N$, then let $A=R[B / b]$ denote the $R$-subalgebra of $R[1 / b]$ generated by the elements $c / b$ with $c \in B$. Let $\mathscr{S}=\mathscr{R}[1 / t b]$. Then $\mathscr{S}=A[t b, 1 / t b]$, and $P^{\prime}=N \mathscr{P} \cap A$ is a prime ideal in $A$. Since $N$ is maximal and isn't homogeneous, $P^{\prime} \mathscr{S} \subset N \mathscr{S}$ and $P=P^{\prime} \mathscr{S} \cap \mathscr{R}$ is a homogeneous prime ideal (as in [13, Remark 3.11]). Also, height $N / P=1$, since $N \mathscr{S} \cap A=P \mathscr{S} \cap A=P^{\prime}$. Since $P$ is homogeneous, (i) and (4.2) imply there exists an $\mathscr{R}$-sequence of $s=$ height $P$ homogeneous elements in $P$, say $x_{1}, \cdots, x_{s}$ (possibly one $x_{h}$ is $u$ ). Since $N$ isn't homogeneous, it follows that $N$ isn't a prime divisor of $\left(x_{1}, \cdots, x_{s}\right) \mathscr{R}$. Also, since $R$ is Macaulay and height $\mathrm{N} / P=1$, height $N=$ height $P+1$ [8, (34.8) and (25.10)]. Hence $\mathscr{R}_{N}$ is Macaulay. 
The next result is a considerable strengthening of [11, Corollary 3.6]. It also shows the unexpected result that every prime ideal $P$ in $R$ contains a prime sequence of height $P$ elements which can be extended to a maximal prime sequence which has the property described in (4.4).

Corollary 4.5. (cf. [11, Corollary 3.6].) Let $\left(R ; M_{1}, \cdots, M_{e}\right), J, B$, $a$, and $\mathscr{R}$ be as in (4.2), assume $\mathscr{R}$ is locally Macaulay, and let $P_{1}$, $\cdots, P_{s}$ be prime ideals in $R$. If height $P_{v}=k(v=1, \cdots, s)$, then there exists an $R$-sequence $y_{1}, \cdots, y_{a}$ such that $y_{1}, \cdots, y_{k}$ are in $\cap P_{v}$ and, for each $j=1, \cdots, a$, for each permutation $\pi$ of $\{1, \cdots, a\}$, for all positive integers $f_{1}, \cdots, f_{k}$, and, for all $n,\left(y_{\pi 1}^{f_{1}}, \cdots, y_{\pi j}^{f_{j}}\right) R \cap B^{n}=$ $\sum_{1}^{j} y_{\pi i}^{f_{i}} B^{n-d_{i} f_{i}}$, where $d_{i}=d_{B}\left(y_{\pi i}\right)$.

Proof. By (4.2), let $x_{1}, \cdots, x_{k}$ be homogeneous elements in $\cap P_{v}^{*}$ such that each permutation of $x_{1}, \cdots, x_{k}, u$ is an $\mathscr{R}$-sequence. By the proof of (4.2), there exist homogeneous elements $x_{k+1}, \cdots, x_{a}$ in $\mathscr{R}$ such that each permutation of $x_{1}, \cdots, x_{a}, u$ is an $\mathscr{R}$-sequence. Hence, for each $j=1, \cdots, a, x_{\pi 1}^{f_{1}}, \cdots, x_{\pi j}^{f_{j}}, u$ is an $\mathscr{R}$-sequence. Let $x_{\pi i}=y_{\pi i} t^{d_{i}}$ with $y_{\pi i} \in B^{d i}(i=1, \cdots, a)$, and, for $j=1, \cdots, a$, let $Y_{j}=$ $\left(y_{\pi 1}^{f_{1}}, \cdots, y_{\pi j}^{f_{j}}\right) R$ and $X_{j}=\left(x_{\pi 1}^{f_{1}}, \cdots, x_{\pi 1}^{f_{j}}\right) \mathscr{R}$. Then $Y_{j *}=X_{j}$, since $X_{j}$ : $u \mathscr{R}=X_{j}$. Hence, since the prime divisors of $Y_{j}^{*}$ are the ideals $P^{*}$ with $P$ a prime divisor of $Y_{j}$, it follows that $y_{\pi 1}^{f_{1}}, \cdots, y_{\pi a}^{f_{a}}$ is an $R$ sequence and, for $j=1, \cdots, a, Y_{j} \cap B^{n}=\left[Y_{j}\right]_{n}=\left[X_{j}\right]_{n}=\sum_{1}^{j} y_{\pi i}^{f_{i}} B^{n-d_{i} f_{i}}$.

The following corollary gives another necessary and sufficient condition for $\mathscr{R}$ to be locally Macaulay.

CoRollary 4.6. With the notation of (4.2), $\mathscr{R}$ is locally Macaulay if and only if there exists a positive integer $g$ and a system of parameters $z_{1}, \cdots, z_{a}$ contained in $B^{g}$ such that, for each $j=1, \cdots, a$, for each (or, for some) permutation $\pi$ of $\{1, \cdots, a\}$, and for all $n \geqq$ $g,\left(z_{\pi 1}, \cdots, z_{\pi j}\right) R \cap B^{n}=\left(z_{\pi 1}, \cdots, z_{\pi j}\right) B^{n-g}$.

Proof. If $\mathscr{R}$ is locally Macaulay, then let $y_{1}, \cdots, y_{a}$ be elements in $J$ as in (4.5). Say $d_{i}=d_{B}\left(y_{i}\right)$, so $d_{i}>0$, by (4.3). Let $D_{i}=\pi_{j \neq i} d_{j}$, let $g=d_{i} D_{i}$, and let $z_{i}=y_{i}^{D i}(i=1, \cdots, a)$. Then the conclusion follows from (4.5). The converse follows from (4.4).

(4.7) and (4.8.1) below are known when $R$ is Macaulay and $B$ is generated by an $R$-sequence [11, Corollary 3.9 and p. 406]. (4.8.2) is new even for the $R$-sequence case but follows easily from (4.7) and (4.8.1); and (4.9) follows from (4.5) - (4.8). The basis of the proof of (4.7) is that, if $b$ is a nonzero-divisor in a locally Macaulay ring $R$, then $R / b R$ is locally Macaulay. 
CoRollary 4.7. (cf. [11, Corollary 3.9].) With the notation of (4.2), if $\mathscr{R}$ is locally Macaulay, then, for each nonzero-divisor $b \in B$, $R\left[b_{1} / b, \cdots, b_{w} / b\right]$ is locally Macaulay, where $B=\left(b_{1}, \cdots, b_{w}\right) R$.

Proof. The proof is the same as the proof of [11, Corollary 3.9].

Corollary 4.8. (cf. [11, p. 406 and Corollary 3.9].) With the notation of (4.2), if $\mathscr{R}$ is locally Macaulay, then the following statements hold, for all positive integers $m$ :

4.8.1. $\mathscr{R}\left(R, B^{m}\right)$ is locally Macaulay.

4.8.2. For each nonzero-divisor $b \in B^{m}, R\left[\beta_{1} / b, \cdots, \beta_{z} / b\right]$ is locally Macaulay, where $B^{m}=\left(\beta_{1}, \cdots, \beta_{z}\right) R$.

Proof. 4.8.1. If $\mathscr{R}$ is locally Macaulay, then $R$ is Macaulay and there exists an $R$-sequence $y_{1}, \cdots, y_{a}$ contained in $J$ such that, for all $j=1, \cdots, a$ and all positive integers $m$ and $n,\left(y_{1}^{m}, \cdots, y_{j}^{m}\right) R \cap B^{n}=$ $\sum_{1}^{j} y_{i}^{m} B^{n-d_{i} m}$, where $d_{B}\left(y_{i}\right)=d_{i}((4.4)$ and (4.5)). Then, with $n=m h$ $(h \geqq 1)$, it follows from (4.4) that $\mathscr{R}\left(R, B^{m}\right)$ is locally Macaulay, if $d_{B^{m}}\left(y_{i}^{m}\right)=d_{i}$. But this holds, since $x_{1}^{m}, \cdots, x_{a}^{m}, u$ is an $\mathscr{R}$-sequence (as in (i) in the proof of (4.4)), where $x_{i}=y_{i} t^{d_{i}}$. (4.8.2) follows from (4.8.1) and (4.7).

Applying the last three corollaries to the case when $R$ is Macaulay and $B$ is a power of the ideal generated by an $R$-sequence, the following corollary is obtained.

CoRollary 4.9. With the notation of (4.2), if $B=Y^{n}$, where $Y$ is generated by an $R$-sequence (such that $\operatorname{Rad} Y=J$ ) and $n>0$, then (4.5) - (4.8) hold.

Proof. This follows from (4.5) - (4.8), since $R$ is Macaulay (since $\operatorname{Rad} Y=J)$, hence $\mathscr{R}$ is locally Macaulay [11, p. 406].

The following proposition has the status of folklore-and may even appear somewhere in the literature. It will be used in (4.11) to prove a number of necessary and sufficient conditions for $\mathscr{R}$ to be locally Macaulay. Also, the relationship, noted below, between Rees rings and form rings together with (4.10) shows that much of the material in this section really isn't so special.

Proposition 4.10. Let $R$ be a Noetherian ring, and let $S$ be a finitely generated positively graded $R$-algebra such that $S_{0}=R$. Then $S$ is locally Macaulay if and only if, for each maximal ideal $M$ in $R . S_{\left(M+S_{+}\right)}$is locally Macaulay, where $S_{+}$is the ideal in $S$ generated 
by the forms of positive degree.

Proof. The condition is clearly necessary. Conversely, let $Q$ be a prime ideal in $S$, and let $M$ be a maximal ideal in $R$ such that $Q \cap R \cong M$. Then $S_{Q}$ is a localization of $R_{M} \otimes_{R} S$, so it may be assumed that $R$ is a local ring with maximal ideal $M$. Let $R(X)=$ $R[X]_{M R[X]}$, where $X$ is an indeterminate, and let $R^{*}$ be the completion of $R(X)$. We can replace $S$ by $R^{*} \otimes_{R} S$, so it may be assumed that $R$ is a complete local ring with an infinite residue field. Then $S$ is a homomorphic image of a regular ring, so the Macaulay locus of $S$ is Zariski open [3, (6.11.3)]. Suppose that the non-Macaulay locus of $S$ isn't empty, and let $I$ be its defining radical ideal. Then it suffices to show that $I$ is homogeneous, for then $I \subseteq M+S_{+}$which contradicts the hypothesis.

If $a \in R, \notin M$, then there exists an $R$-automorphism of $S$ which takes each form $F$ of degree $d$ to $a^{d} F$. Therefore, let $\sum_{0}^{d} F_{i} \in I$ (where each $F_{i}$ is a form of degree $i$ ) and choose units $a_{0}, \cdots, a_{d}$ in $R$ with distinct residue classes modulo $M(R / M$ is infinite). Then, since clearly $I$ is invariant under every automorphism on $S, \sum_{i=0}^{d} a_{j}^{i} F_{i}$ is in $I(0 \leqq j$ $\leqq d)$. But Det $\left(a_{j}^{j}\right)= \pm \pi_{i<j}\left(a_{i}-a_{j}\right) \in R$, $\notin M$, hence is a unit in $R$. Therefore each $F_{i} \in I$, as desired.

If $B$ is an ideal in a Noetherian ring $R$, then, as in [15, Theorem 2.1], the form ring $\mathscr{F}=\mathscr{F}(R, B)$ of $R$ with respect to $B$ is (isomorphic to) $\mathscr{R} / u \mathscr{R}$, and the $B$-form ideal $C^{\prime}$ of an ideal $C$ in $R$ is (isomorphic to) $\left(C^{*}, u\right) \mathscr{R} / u \mathscr{R}$. This fact is used in (4.11) below.

If $M_{1}, \cdots, M_{e}$ are special maximal ideals in a Noetherian ring $R$ such that each $R_{M_{i}}$ is Macaulay, then it isn't true, in general, that $R$ is locally Macaulay. However, this is true for $\mathscr{R}$ and $\mathscr{F}$, as is shown by the following theorem.

Theorem 4.11. Let $B$ be an ideal in a Noetherian ring $R$ such that $B$ is contained in the Jacobson radical of $R$, let $\mathscr{R}=\mathscr{R}(R, B)$, and let $\mathscr{F}=\mathscr{F}(R, B)$ be the form ring of $R$ with respect to $B$. Then statements (4.11.1)-(4.11.4) below are equivalent and each implies (4.11.5).

4.11.1. $\mathscr{R}$ is locally Macaulay.

4.11.2. $\mathscr{R}_{\mathbb{N}}$ is Macaulay, for all maximal homogeneous ideals th in $\mathscr{R}$.

4.11.3. $\mathscr{F}$ is locally Macaulay.

4.11.4. $\mathscr{F}_{\mathscr{r}}$ is Macaulay, for all maximal homogeneous ideals $\mathscr{N}$ in $\mathscr{F}$.

4.11.5. $R$ and all rings $R\left[b_{1} / b, \cdots b_{w} / b\right]$ are locally Macaulay, where $b$ is a nonzero-divisor in $B=\left(b_{1}, \cdots, b_{w}\right) R$. 
Proof. Clearly (4.11.1) implies (4.11.2), and (4.11.3) implies (4.11.4). Also, (4.11.1) implies (4.11.3), and (4.11.2) implies (4.11.4), since $\mathscr{F} \cong \mathscr{R} / u \mathscr{R}$ (as in [15, Theorem 2.1]). Further, (4.11.4) implies (4.11.3), by (4.10). Now (4.11.3) implies $\mathscr{R}_{P}$ is Macaulay, for all prime ideals $P$ in $\mathscr{R}$ such that $u \in P$. Thus, if $M$ is a maximal ideal in $R$, then $\mathscr{C}=\left(M^{*}, u\right) \mathscr{R}$ is a maximal ideal in $\mathscr{R}$ (since $B \subseteq M)$, so, since $\mathscr{R}_{\mathscr{N}}$ is Macaulay, $\mathscr{R}_{M^{*}}=R[u]_{M R[u]}$ is Macaulay. It follows that $R$ is locally Macaulay, and so $R[t, u]$ is locally Macaulay. From this it follows that (4.11.3) implies (4.11.1), and so (4.11.1)(4.11.4) are equivalent and each implies that $R$ is locally Macaulay. Finally, since $R\left[b_{1} / b, \cdots, b_{w} / b\right][t b, 1 / t b]=\mathscr{R}[1 / t b]$ and $t b$ is transcendental over $R$, (4.11.1) implies (4.11.5).

This paper will be closed with the following result which gives two equivalences of (4.11.5).

Proposition 4.12. Let $B$ be an ideal in a Noetherian ring $R$ such that $B$ is contained in the Jacobson radical of $R$, and let $\mathscr{R}=$ $\mathscr{R}(R, B)$. Then the following statements are equivalent:

4.12.1. $R$ and all rings $R\left[b_{1} / b, \cdots, b_{w} / b\right]$ are locally Macaulay, where $B=\left(b_{1}, \cdots, b_{w}\right) R$ and $b$ is a nonzero-divisor in $B$.

4.12.2. For each prime ideal $P$ in $\mathscr{R}$ such that $\left(u, B^{*}\right) \nsubseteq P, \mathscr{R}_{P}$ is Macaulay.

4.12.3. For each homogeneous prime ideal $P$ in $\mathscr{R}$ such that $\left(u, B^{*}\right) \mathscr{R} \nsubseteq P, \mathscr{R}_{P}$ is Macaulay.

Proof. If $K$ is a ring and $X$ an indeterminate, then $K$ is locally Macaulay if and only if $K[X, 1 / X]$ is locally Macaulay. The equivalence of (4.12.1) and (4.12.2) follows from this and the facts $\mathscr{R}[1 / u]=R[t, u]$ and $\mathscr{R}[1 / t b]=A[t b, 1 / t b]$, where $A=R\left[b_{1} / b, \cdots, b_{w} / b\right]$. Clearly (4.12.2) implies (4.12.3). Also, $R[t, u]_{M R[t, u]}=\mathscr{R}_{M^{*}}$ (where $M$ is a maximal ideal in $R$ ), and, if $P^{\prime}$ is a prime ideal in $A$, then $P=P^{\prime} \mathscr{R}[1 / t b] \cap \mathscr{R}$ is homogeneous (as in [13, Remark 3.11]) and $A[t b]_{P^{\prime} A[t b]}=\mathscr{R}_{P}$. Therefore, (4.12.3) implies (4.12.1).

\section{REFERENCES}

1. D. Ferrand and M. Raynaud, Fibres formelles d'un anneau local Noetherian, Ann. Sci. École Norm. Sup., 3 (1970), 295-311.

2. A. Grothendieck, Elements de Geometrie Algebrique, IV (Première Partie), Paris: Institut des Hautes Etudes Scientifiques, 1964.

3. - Elements de Geometrie Algebrique, IV (Seconde Partie), Paris: Institut des Hautes Etudes Scientifiques, 1965.

4. ——, Elements de Geometrie Algebrique, IV (Troisième Partie), Paris: Institut des Hautes Etudes Scientifiques, 1966.

5. I. Kaplansky, Commutative Rings, Boston: Allyn and Bacon, 1970. 
6. S. McAdam, Saturated Chains in Noetherian rings, preprint.

7. M. Nagata, On the chain problem of prime ideals, Nagoya Math. J., 10 (1956), 51-64.

8. — Local Rings, New York: Interscience, 1962.

9. D. G. Northcott, Lessons on Rings, Modules, and Multiplicities, London: Cambridge University Press, 1968.

10. L. J. Ratliff, Jr., On quasi-unmixed semi-local rings and the altitude formula, Amer. J. Math., 87 (1965), 278-284.

11. - Two notes on locally Macaulay rings, Trans. Amer. Math. Soc., 119 (1965), 399-406.

12. - On quasi-unmixed local domains, the altitude formula, and the chain condition for prime ideals (I), Amer. J. Math., 91 (1969), 508-528.

13. - On quasi-unmixed local domains, the altitude formula, and the chain condition for prime ideals (II), Amer. J. Math., 92 (1970), 99-144.

14. - Characterizations of catenary rings, Amer. J. Math., 93 (1971), 1070-1108.

15. D. Rees, $A$ note on form rings and ideals, Mathematika, 4 (1957), 51-60.

16. D. Rees, $\mathfrak{A}$-transforms of local rings and a theorem on multiplicities of ideals, Proc. Cambridge Philos. Soc., 57 (1961), 8-17.

17. O. Zariski and P. Samuel, Commutative Algebra, Vol II, New York: D. Van Nostrand, 1960.

Received September 24, 1971 and in revised form August 21, 1972. Work on this paper was supported in part by the National Science Foundation: The first author by Grant GP-29224X and the second by Grant NSF-28939.

UNIVERSITY OF MINNESOTA

AND

UNIVERSITY OF CALIFORNIA, RIVERSIDE 


\section{PACIFIC JOURNAL OF MATHEMATICS}

\section{EDITORS}

H. SAMELSON

Stanford University

Stanford, California 94305

C. R. Hовву

University of Washington

Seattle, Washington 98105
J. DugundJI

Department of Mathematics University of Southern California

Los Angeles, California 90007

RICHARD ARENS

University of California

Los Angeles, California 90024

\section{ASSOCIATE EDITORS}

E. F. BECKENBACH

B. H. NeumanN

F. WOLF

K. YoSHIDA

\section{SUPPORTING INSTITUTIONS}

UNIVERSITY OF BRITISH COLUMBIA

CALIFORNIA INSTITUTE OF TECHNOLOGY

UNIVERSITY OF CALIFORNIA

MONTANA STATE UNIVERSITY

UNIVERSITY OF NEVADA

NEW MEXICO STATE UNIVERSITY

OREGON STATE UNIVERSITY

UNIVERSITY OF OREGON

OSAKA UNIVERSITY
UNIVERSITY OF SOUTHERN CALIFORNIA

STANFORD UNIVERSITY

UNIVERSITY OF TOKYO

UNIVERSITY OF UTAH

WASHINGTON STATE UNIVERSITY

UNIVERSITY OF WASHINGTON

AMERICAN MATHEMATICAL SOCIETY

NAVAL WEAPONS CENTER

Printed in Japan by International Academic Printing Co., Ltd., Tokyo, Japan 


\section{Pacific Journal of Mathematics}

\section{Vol. 44, No. $1 \quad$ May, 1973}

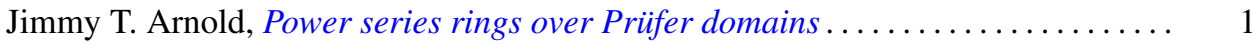

Maynard G. Arsove, On the behavior of Pincherle basis functions . . . . . . . . . 13

Jan William Auer, Fiber integration in smooth bundles ................. 33

George Bachman, Edward Beckenstein and Lawrence Narici, Function algebras

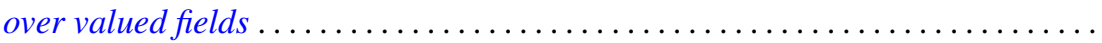

Gerald A. Beer, The index of convexity and the visibility function . . . . . . . . . . .

James Robert Boone, A note on mesocompact and sequentially mesocompact

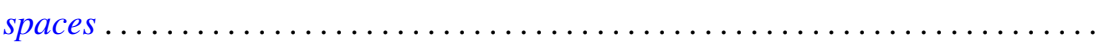

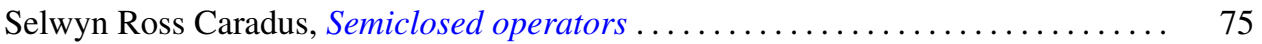

John H. E. Cohn, Two primary factor inequalities . . . . . . . . . . . . . . . 81

Mani Gagrat and Somashekhar Amrith Naimpally, Proximity approach to

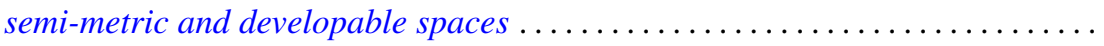

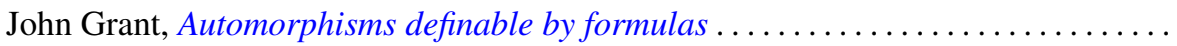

Walter Kurt Hayman, Differential inequalities and local valency ..............

Wolfgang H. Heil, Testing 3-manifolds for projective planes . . . . . . . . . . . . .

107

Melvin Hochster and Louis Jackson Ratliff, Jr., Five theorems on Macaulay

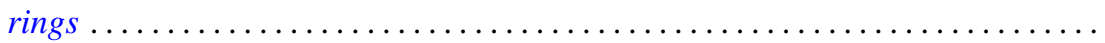

Thomas Benton Hoover, Operator algebras with reducing invariant subspaces ....

James Edgar Keesling, Topological groups whose underlying spaces are separable

Fréchet manifolds...

Frank Leroy Knowles, Idempotents in the boundary of a Lie group . .

191

George Edward Lang, The evaluation map and EHP sequences ...

201

Everette Lee May, Jr, Localizing the spectrum . . . . . . . . . . . .

211

Frank Belsley Miles, Existence of special $K$-sets in certain locally compact abelian groups.

Susan Montgomery, A generalization of a theorem of Jacobson. II . .

T. S. Motzkin and J. L. Walsh, Equilibrium of inverse-distance forces in

three-dimensions.

Arunava Mukherjea and Nicolas A. Tserpes, Invariant measures and the converse

of Haar's theorem on semitopological semigroups .

James Waring Noonan, On close-to-convex functions of order $\beta$

Donald Steven Passman, The Jacobian of a growth transformation

Dean Blackburn Priest, A mean Stieltjes type integral ........ .

Joe Bill Rhodes, Decomposition of semilattices with applications to topological

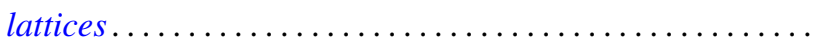

Claus M. Ringel, Socle conditions for $\mathrm{QF}-1$ rings ..........

Richard Rochberg, Linear maps of the disk algebra

Roy W. Ryden, Groups of arithmetic functions under Dirichlet convolution . .

Michael J. Sharpe, A class of operators on excessive functions

Erling Stormer, Automorphisms and equivalence in von Neumann algebras ..

Philip C. Tonne, Matrix representations for linear transformations on series

analytic in the unit disc. 